\title{
Identification of new QTLs for resistance to Plasmodiophora brassicae in Brassica napus using genome wide association mapping
}

\section{Abdulsalam Dakouri}

Saskatoon Research and Development Centre

Mebarek Lamara

Saskatoon Research and Development Centre

Md. Masud Karim

Saskatoon Research and Development Centre

Jinghe Wang

Saskatoon Research and Development Centre

\section{Qilin Chen}

Saskatoon Research and Development Centre

\section{Stephen E. Strelkov}

University of Alberta

\section{Sheau-Fang Hwang}

University of Alberta

Bruce D. Gossen

Saskatoon Research and Development Centre

Gary Peng

Saskatoon Research and Development Centre

Fengqun Yu ( $\square$ fengqun.yu@canada.ca)

Agriculture and Agri-Food Canada https://orcid.org/0000-0002-7957-7632

\section{Research article}

Keywords: Brassica napus, Plasmodiophora brassicae, genetic resistance, genome-wide association mapping, resistance

Posted Date: September 12th, 2019

DOI: https://doi.org/10.21203/rs.2.14300/v1

License: (a) (i) This work is licensed under a Creative Commons Attribution 4.0 International License. Read Full License 


\section{Abstract}

Background Clubroot of canola (Brassica napus ), caused by the obligate pathogen Plasmodiophora brassicae Woronin, is a major disease worldwide. Genetic resistance remains the best strategy to manage this disease. The objective of the study was to identify and map new sources of resistance to clubroot in B. napus using genome-wide association mapping. The reaction of a collection of 177 accessions to four highly virulent pathotypes of P. brassicae was assessed. These pathotypes were selected because they were most recently identified and showed different virulence patterns on the Canadian clubroot differential (CCD) lines. The collection was then genotyped using genotyping by sequencing (GBS) method. Multi-locus mixed linear model (MMLM) was used to perform the association analysis.

Results The majority of accessions were highly susceptible (70 -100 DSI), while few individual accessions showed strong resistance (0-20 DSI) to $5 \mathrm{X}$ (2 accessions), 2B (7 accessions), 3A (8 accessions) and 3D (15 accessions). In total, 301,753 SNPs were mapped to 19 chromosomes. Population structure analysis indicated that the 177 accessions belong to two major populations. SNPs were associated with resistance to each pathotype using MLMM. In total, 23 significant SNP loci were identified, with 14 SNPs mapped to the A-genome and 9 to the C-genome. The SNPs were associated with resistance to pathotypes $5 X$ (4 SNPs), 2B (9), 3A (5) and 3D (5). A blast search of $2 \mathrm{Mb}$ upstream and downstream identified 61 disease resistance genes, of which 24 belonged to TIR-NBS-LRR proteins and 20 belonged to CC-NBS-LRR proteins. The distance between a SNP locus and the nearest resistance genes ranged from $0.11-1.66 \mathrm{Mb}$. This indicated that NBS-LRR gene family might have an important role in clubroot resistance in B. napus .

Conclusion The resistant B. napus lines and the SNP markers identified in this study can be used for breeding for resistance to clubroot and contribute to understanding the genetic mechanism of resistance to clubroot.

\section{Background}

Canola (Brassica napus L.), also known as oilseed rape, is grown around the globe [1]. Canola is the largest crop in Canada by acreage (22,535,000 acres) and farm gate value ( $\$ 16.7$ billion $\mathrm{Cdn})$. Demand for a healthy oil for human consumption, biofuel production, and use of meal as a high quality feed for livestock, have produced strong prices and steadily increasing canola production (Canola Council of Canada 2019).

Brassica napus (AACC, $2 n=38$ ), is a natural amphidiploid species resulting from several hybridization events between its progenitors; $B$. rapa L. (AA genome, $2 n=20$ ) and $B$. oleracea $L$. (CC genome, $2 n=18$ ) [2]. The $A$ and $C$ genomes remained distinct, so recombination events are rare and there is no chromosomal rearrangement $[3,4]$. There are two forms of $B$. napus; biannual vegetables such as rutabaga and swede, and annual oilseed or fodder subspecies that contains many economically important oil, leafy and root vegetables, and fodder crops [5]. 
Clubroot, caused by Plasmodiophora brassicae Woronin, is an important disease of canola/oilseed rape and other brassica species worldwide [6,7]. Management based on genetic resistance has been effective $[8,9]$ but is generally not durable. The clubroot resistance (CR) genes identified have been almost exclusively from $B$. rapa $[10,11]$. Nonetheless, a few resistant $B$. napus lines have been used as sources of CR genes [12,13].

Linkage mapping has been used extensively to study important qualitative and quantitative traits, but it is limited to detecting pairs of alleles representing the two parents of the mapping population [14]. Association mapping of population based on linkage disequilibrium (LD) between alleles within diverse populations can be used to detect potential association between markers and traits of interest [15]. The result of advances in next-generation sequencing technologies is that analysis of genotype by sequencing has become feasible for plant species with large genome size [16].

Association mapping has been used in various host-pathogen systems including wheat [17], tomato [18], maize [19] and canola $[13,20]$. Genome-wide association mapping in $B$. napus-P. brassicae has been limited to one study using already established SNP array and a single pathotype [13].

In the current study, a world collection of 177 B. napus germplasm was genotyped by GBS approach and tested for their reaction to four very recently identified pathotypes of $P$. brassicae. These pathotypes exhibited various virulence patterns on the Canadian clubroot differential (CCD) lines. The objective was to identify novel sources of resistance to clubroot from this large collection of $B$. napus accessions by (1) screening the collection under controlled environment, (2) assessing genetic diversity and structure analysis of the core collection, and (3) conducting association mapping of resistance to clubroot in this collection.

\section{Full Text}

Identification of new QTLs for resistance to Plasmodiophora brassicae in Brassica napus using genome wide association mapping

Abdulsalam Dakouri ${ }^{1}$, Mebarek Lamara ${ }^{1,2}$, Md. Masud Karim ${ }^{1}$, Jinghe Wang ${ }^{1}$, Qilin Chen ${ }^{1}$, Stephen E. Strelkov ${ }^{3}$, Sheau-Fang Hwang ${ }^{3}$, Bruce D. Gossen ${ }^{1}$, Gary Peng ${ }^{1}$, Fengqun $\mathrm{Yu}^{1}$

${ }^{1}$ Saskatoon Research and Development Centre, Agriculture and Agri-Food Canada, Saskatoon, SK S7N 0X2, Canada.

${ }^{2}$ Institut de Recherche sur les Forêts, Université du Québec en Abitibi-Témiscamingue, Rouyn-Noranda, QC J9X 5E4, Canada.

${ }^{3}$ Department of Agricultural, Food and Nutritional Science, University of Alberta, Alberta, Canada.

*Corresponding author: Fengqun Yu. Email: fengqun.yu@canada.ca 


\section{Emails address of authors}

Abdulsalam Dakouri : abdulsalam.dakouri@canada.ca

Mebarek Lamara: mebarek.lamara@uqat.ca

Md. Masud Karim: masud.karim@canada.ca

Jinghe Wang: jinghe.wang@canada.ca

Qilin Chen: qilin.chen@canada.ca

Bruce Gossen: bruce.gossen@canada.ca

Stephen E. Strelkov: strelkov@ualberta.ca

Sheau-Fang Hwang: sh20@ualberta.ca

Gary Peng: gary.peng@canada.ca

Fengqun Yu: fengqun.yu@canada.ca

\section{Abstract}

\section{Background}

Clubroot of canola (Brassica napus)," caused by the obligate pathogen Plasmodiophora brassicae Woronin, is a major disease worldwide. Genetic resistance remains the best strategy to manage this disease. The objective of the study was to identify and map new sources of resistance to clubroot in $B$. napus using genome-wide association mapping. The reaction of a collection of 177 accessions to four highly virulent pathotypes of $P$. brassicae was assessed. These pathotypes were selected because they were most recently identified and showed different virulence patterns on the Canadian clubroot differential (CCD) lines. The collection was then genotyped using genotyping by sequencing (GBS) method. Multi-locus mixed linear model (MMLM) was used to perform the association analysis.

\section{Results}

The majority of accessions were highly susceptible (70-100 DSI), while few individual accessions showed strong resistance (0-20 DSI) to 5X (2 accessions), 2B (7 accessions), 3A (8 accessions) and 3D (15 accessions). In total, 301,753 SNPs were mapped to 19 chromosomes. Population structure analysis indicated that the 177 accessions belong to two major populations. SNPs were associated with resistance to each pathotype using MLMM. In total, 23 significant SNP loci were identified, with 14 SNPs 
mapped to the A-genome and 9 to the $\mathrm{C}$-genome. The SNPs were associated with resistance to pathotypes 5X (4 SNPs), 2B (9), 3A (5) and 3D (5). A blast search of $2 \mathrm{Mb}$ upstream and downstream identified 61 disease resistance genes, of which 24 belonged to TIR-NBS-LRR proteins and 20 belonged to CC-NBS-LRR proteins. The distance between a SNP locus and the nearest resistance genes ranged from 0.11-1.66 Mb. This indicated that NBS-LRR gene family might have an important role in clubroot resistance in $B$. napus.

\section{Conclusion}

The resistant $B$. napus lines and the SNP markers identified in this study can be used for breeding for resistance to clubroot and contribute to understanding the genetic mechanism of resistance to clubroot.

Keywords: Brassica napus, Plasmodiophora brassicae, genetic resistance, genome-wide association mapping, resistance.

\section{Background}

Canola (Brassica napus L.), also known as oilseed rape, is grown around the globe [1]. Canola is the largest crop in Canada by acreage (22,535,000 acres) and farm gate value ( $\$ 16.7$ billion Cdn). Demand for a healthy oil for human consumption, biofuel production, and use of meal as a high quality feed for livestock, have produced strong prices and steadily increasing canola production (Canola Council of Canada 2019).

Brassica napus (AACC, $2 n=38$ ), is a natural amphidiploid species resulting from several hybridization events between its progenitors; $B$. rapa $L$. (AA genome, $2 n=20)$ and $B$. oleracea $L$. (CC genome, $2 n=18$ ) [2]. The $A$ and $C$ genomes remained distinct, so recombination events are rare and there is no chromosomal rearrangement [3,4]. There are two forms of $B$. napus; biannual vegetables such as rutabaga and swede, and annual oilseed or fodder subspecies that contains many economically important oil, leafy and root vegetables, and fodder crops [5].

Clubroot, caused by Plasmodiophora brassicae Woronin, is an important disease of canola/oilseed rape and other brassica species worldwide [6,7]. Management based on genetic resistance has been effective $[8,9]$ but is generally not durable. The clubroot resistance (CR) genes identified have been almost exclusively from $B$. rapa $[10,11]$. Nonetheless, a few resistant $B$. napus lines have been used as sources of CR genes [12,13].

Linkage mapping has been used extensively to study important qualitative and quantitative traits, but it is limited to detecting pairs of alleles representing the two parents of the mapping population [14]. Association mapping of population based on linkage disequilibrium (LD) between alleles within diverse populations can be used to detect potential association between markers and traits of interest [15]. The 
result of advances in next-generation sequencing technologies is that analysis of genotype by sequencing has become feasible for plant species with large genome size [16].

Association mapping has been used in various host-pathogen systems including wheat [17], tomato [18], maize [19] and canola $[13,20]$. Genome-wide association mapping in $B$. napus- $P$. brassicae has been limited to one study using already established SNP array and a single pathotype [13].

In the current study, a world collection of 177 B. napus germplasm was genotyped by GBS approach and tested for their reaction to four very recently identified pathotypes of $P$. brassicae. These pathotypes exhibited various virulence patterns on the Canadian clubroot differential (CCD) lines. The objective was to identify novel sources of resistance to clubroot from this large collection of $B$. napus accessions by (1) screening the collection under controlled environment, (2) assessing genetic diversity and structure analysis of the core collection, and (3) conducting association mapping of resistance to clubroot in this collection.

\section{Results}

\section{Evaluation of clubroot reaction}

At 6 weeks after seeding, the germplasm was evaluated for resistance to four $P$. brassicae pathotypes; $5 \mathrm{X}$, $2 \mathrm{~B}, 3 \mathrm{~A}$ and $3 \mathrm{D}$. The disease severity index (DSI) ranged from 0 to 100 . The majority of accessions were highly to completely susceptible (70-100 DSI), but several were highly resistant (0-20 DSI) to pathotypes 5X (21 accessions), 2B (7 accessions), 3A (8 accessions), and 3D (15 accessions) (Fig. 1, Table S1). The correlation coefficient of severity among the four pathotypes was strongest between $2 \mathrm{~B}$ and $3 \mathrm{~A}\left(r^{2}=\right.$ $0.77)$ and weakest between $5 \mathrm{X}$ and 3D $\left(r^{2}=0.27\right.$, Table S2). Phenotypic data were transformed using rank-based inverse normal transformation to make the DSI values nearly fit the normal distribution required for parametric model-based association analysis (Figure S1).

\section{Sequence analysis and SNP discovery}

Genotyping by sequencing (GBS) data analysis was performed for the 177 B. napus accessions. A total of $\sim 1.2$ billion reads and $\sim 633$ million good barcoded reads were generated and split into three FASTQ fills. On average, there were 3.3 $\mathrm{M}$ read counts per sample (range $\sim 1.8$ to $7.7 \mathrm{M}$ ) and $3.1 \mathrm{M}$ read counts mapped (range 76 to $96 \%$ ). Sequence tags from each file were captured and merged to produce a master tag file of 4,253,499 sequence tags. The tags were then aligned to B. napus reference genome v4.1, using the TASSEL-GBS pipeline. A total of 2,217,292 (52.1\%) tags were uniquely aligned to the reference, $1,220,090(28.7 \%)$ aligned to multiple positions and 816,117 (19.2\%) were not aligned. Uniquely mapped tags were used to calculate the tag density distribution at each site in the B. napus genome and for SNP calling. 
The raw sequence data for SNP calling were also analysed using the TASSEL-GBS pipeline. A total of 399,234 unfiltered SNPs and 355,680 filtered SNPs were called for the 177 accessions, with a mean of individual depth of $8.5 \pm 2$ SD and mean site depth of $6.7 \pm 11.4$ SD. Of the 355,680 filtered SNPs, 301,753 SNPs were mapped to the 19 chromosomes; the remaining SNPs were randomly distributed without specific chromosome assignment. Only variants mapped to chromosomes were kept for further analyses.

\section{Variant analysis and annotation}

There were more SNPs in the C-genome (160,174 SNPs) than the A-genome (141,579 SNPs). Chromosome $\mathrm{A} 03$ had the highest number of SNPs within the A-genome, while $\mathrm{C03}$ contained the highest number of SNPs in the C-genome (Table 1). The mean density per Kb was 2.12 SNP / Kb across the 19 chromosomes. In general, SNP density was higher in the C- genome (2.55 SNPs / Kb) than the A-genome (1.70 SNPs / Kb). C07 had the highest number of SNPs per Kb (2.88) and A10 had the lowest (1.43) (Table 1). The vast majority of SNPs were bi-allelic (90\%), and only $10 \%$ were multi-allelic (Figure S2). There was a positive correlation $\left(r^{2}=0.80\right)$ between chromosome length and the number of SNPs, but only a weak correlation $\left(r^{2}=0.3\right)$ between the number of SNPs and the number of SNPs per Kb.

The SNPs were annotated using the VariantAnnotation package of R. About $37 \%$ of SNPs were annotated within coding regions, $22 \%$ within introns, $31 \%$ within promoter regions, $0.3 \%$ within splice sites, and $9.7 \%$ mapped to other genetic regions (Figure S3). A more detailed SNP annotation was performed using the Variant Effect Predictor (Figure S3). For SNPs within coding regions, 17\% were non-synonymous, 18\% were upstream-gene variants, $9 \%$ were downstream-gene variants, $23 \%$ were synonymous variants, $14 \%$ were intron variants, $15 \%$ intergenic variants, and $4 \%$ were located in the splice site regions and $5^{\prime}$ and $3^{\prime}$ UTRs (Figure S2C). Overall, more SNPs were annotated to the A-genome than the C-genome (Figure S3).

\section{Genetic diversity and population structure}

For genetic diversity analysis, the SNP markers were filtered at a minor allele frequency (MAF) of 0.05 and minimum sample count of $80 \%$, which resulted in 140,195 good quality SNPs. The mean MAF was the same for the $A$ - and $\mathrm{C}$-genomes $(\mathrm{MAF}=0.14)$. Chromosome $\mathrm{C} 01$ had the highest MAF $(0.16)$, followed by $\mathrm{C} 03$ and A07 (0.15), and lowest in chromosomes A09 and C09 (0.12) (Table 1). The mean marker heterozygosity $\left(\mathrm{H}_{\mathrm{e}}\right)$ was 0.06 and the mean accession heterozygosity was 0.14 . The average polymorphic information content (PIC) was the same for A and C-genomes (0.26). PIC was highest in chromosome C01 (0.27) and lowest (0.24) in A09 (Table 1). The ratio of transitions (changes from $A<->$ and $C<->$ ) to transversions (changes from $A$ $<>C, A<->, G<->$ or $G<->T$ ) was 3.22.

Population structure analysis indicated the existence of two major group populations, and analysis using the Evanno criterion supported this result (Fig. 2). Population 1 contained 63 accessions (35.6\%) representing all continents, while population 2 contained 114 accessions (64.4\%), mainly from Europe. A 
phylogenetic tree using the neighbour-joining algorithm produced two major clusters and six subclusters (Figure S4).

\section{Analysis of molecular variance}

Analysis of molecular variance on the six subclusters (SCA-I, II, III,SCB-I, II and III) identified significant genetic differences between major clusters, among subclusters, and among individuals within subclusters $(p<0.001)$. Variance within subclusters accounted for $87.7 \%$ of the total variance, with only $7.5 \%$ among sub-cluster and $4.7 \%$ among major clusters. The fixation index $\left(F_{s t}\right)$ value was 0.21 , which indicated that the accessions belonged to two closely related groups (Table S3). Sub-cluster pairwise $F_{\text {st }}$ values ranged from 0.03 between SCB-I and SCB-II to 0.16 between SCA-I and SCB-II (Table S4).

\section{Linkage disequilibrium analysis}

Linkage disequilibrium in the association panel was calculated using Pearson's $r^{2}$ statistic on pairwise combinations of SNPs present across the 19 chromosomes of B. napus (Figure S5). The average LD ( $\left.r^{2}\right)$ across the genome was 0.15. The mean LD was 0.10 in the A-genome and 0.19 in the $C$-genome. LD values ranged from 0.01 in $\mathrm{A} 09$ to 0.19 in $\mathrm{C} 01$ (Table 1). Across the genome, LD decayed very rapidly $\left(r^{2}=\right.$ 0.20) within $300 \mathrm{~Kb}$ (Figure S5).

\section{Association analysis}

Genome-wide association analysis for clubroot severity was conducted using the following models: general linear model (GLM), mixed linear model (MLM), compressed mixed linear model (CMLM), enriched compressed mixed linear model (ECMLM), and multi-locus mixed model (MLMM). The quantilequantile (Q-Q) plots, from all models revealed that, save for significant SNPs, the distribution of observed $-\log 10(p)$ was closest to the expected distribution in the MLMM compared to other models, therefore associations were identified using this model. A significance threshold of $P<0.5 / \mathrm{N}$ (N: number of SNPs) was used for detecting significant SNPs. The MLMM-genome-wide association study (GWAS) detected 23 SNPs associated with resistance to the four $P$. brassicae pathotypes including four SNPs associated with resistance to $5 \mathrm{X}$, nine SNPs to $2 \mathrm{~B}$, five to $3 \mathrm{~A}$ and five to $3 \mathrm{D}$. The name, physical position, $P$ value and $-\log (P$ value $)$ are presented in Table 2. Across genome, the A-genome carried 14 SNP loci and the Cgenome carried 11 loci (Table 2, Fig. 3).

\section{Candidate resistance genes}

A Blast search identified 61 nucleotide binding site/leucine-rich repeat (NBS-LRR) resistance proteins and non-NBS-LRR resistance genes within the $2 \mathrm{Mb}$ sequence upstream and downstream of 19 out of 23 
significant SNP loci detected in our study (Table 3). The majority of resistance genes appeared as clusters of 2 to 10 genes, while they appeared as a single gene in other cases. On A01, one resistance gene (BnaA01g28560D) was found at $\sim 0.5 \mathrm{Mb}$ from the A01_19406286 locus associated with resistance to pathotype 5X. On A03, one Enhanced Disease Resistance 2-like (BnaA03g03110D) gene and two TIRNBS-LRR resistance (BnaA03g03260D, BnaA03g03270D) genes were detected at 0.11-0.2 Mb distance from A03_651104541 locus associated with resistance to pathotype 2B. Additionally, a cluster of two TIRNBS-LRR resistance (BnaA03g44070D, BnaA03g44080D) genes and a Disease Resistance RRS1-like isoform $\mathrm{X} 1$ were detected at $0.73 \mathrm{Mb}$ distance from $A 03 \_68863700$ locus associated with resistance to pathotype 2B (Table 3). A cluster of six TIR-NBS-LRR resistance (BnaA03g45000D, BnaA03g45010D, BnaA03g45020D, BnaA03g45040D, BnaA03g45050D) genes were identified on A03 at 0.11-0.13 Mb from the A03_71057307 locus associated with resistance to pathotype 3D (Table 3). On A04, a gene (BnaA04g06780D) encoding a Disease Resistance-Responsive (dirigent-like protein) protein family and four putative disease resistance genes (BnaA04g06520D, BnaA04g06530D, BnaA04g06550D, BnaA04g06580D) were identified at 0.63-0.92 Mb from A04_83864566 locus associated with resistance to 2B. On A05, two CC-NBS-LRR (BnaA05g24990D, BnaA05g25000D) genes were identified at $\sim 0.2 \mathrm{Mb}$ from A05_115772286 locus associated with resistance to pathotype 2B. On A08, one disease resistance gene (BnaA08g02210D) was detected at $0.94 \mathrm{Mb}$ from A08_171171159 locus associated with resistance to pathotype 5X. Also, a cluster of six CC-NBS-LRR genes were found at 0.12-0.52 Mb from A08_186203638 associated with resistance to 2B. On A09, a cluster of five TIR-NBS-LRR resistance genes (BnaA09g13280D, BnaA09g13850D, BnaA09g13890D, BnaA09g13900D, BnaA09g14320D) and five CCNBS-LRR genes (BnaA09g14420D, BnaA09g14550D, BnaA09g14560D, BnaA09g14570D, BnaA09g14580D) were detected at 0.11-0.76 Mb from A09_195497763 locus associated with resistance to 5X. In addition, one CC-NBS-LRR gene (BnaA09g42680D),, two Disease Resistance-Responsive genes (dirigent-like protein) and two Enhanced Disease Resistance 4-like genes were found at 0.50-1.67 Mb from A09_215839211 locus associated with resistance to pathotype 2B. On A10, one enhanced disease resistance-like gene (BnaA10g04000D), , and one CC-NBS-LRR gene (BnaA10g05000D) were detected at 0.98-1.50 Mb from the A10_222415846 locus associated with resistance to pathotype 3A.

On C01, four TIR-NBS-LRR resistance genes (BnaC01g40270D, BnaC01g40280D, BnaC01g40300D, BnaC01g40310D) and two non-NBS-LRR disease resistance genes (BnaC01g39050D, BnaC01g40460D) were identified at $0.36-0.46 \mathrm{Mb}$ from the locus at C01_276968702 associated with resistance to pathotype 3A (Table 3). On C03, two TIR-NBS-LRR resistance genes (BnaC03g05380D, BnaC03g04690D) located at $0.16 \mathrm{Mb}$ and $0.48 \mathrm{Mb}$ from C03_68899547 and C03_685453245 loci associated with resistance to pathotype 3D were detected. On C04, one TIR-NBS-LRR resistance gene (BnaC08g17450D) and one CC-NBS-LRR resistance gene (BnaC04g18730D) mapped at $0.31 \mathrm{Mb}$ and $0.48 \mathrm{Mb}$, respectively, from the C04_403341747 locus associated with resistance to pathotype 3A (Table 3). On C08, one TIRNBS-LRR resistance (BnaC08g17450D) gene was identified at $0.31 \mathrm{Mb}$ from C08_579178095 locus associated with resistance to 5X. On C09, two TIR-NBS-LRR resistance genes (BnaC09g 14400D, BnaC09g14870D) and three CC-NBS-LRR resistance genes (BnaC09g15010D, BnaC09g15020D, BnaC09g15110D) located at 0.12-0.4 Mb from C09_608174205 locus associated with resistance to $3 \mathrm{~A}$. 
In addition, a non-NBS-LRR disease resistance gene (BnaC09g38250D) located at $0.38 \mathrm{Mb}$ from C09_638459286 locus associated with resistance to 3D was detected (Table 3).

\section{Discussion}

GWAS has been widely used to identify and map QTLs for quantitatively inherited traits in a wide range of plant species. In the current study, GWAS was used to identify and map new sources of resistance to four highly aggressive pathotypes (5X,2B, 3A, 3D) of $P$. brassicae in 177 accessions of $B$. napus. The majority of the accessions were highly susceptible to all four pathotypes (80-100 DSI), while 10\% showed high levels of resistance (0-25 DSI). This supported previous reports that sources of high levels of resistance to clubroot were much less common in B. napus than in B. rapa [11,12]. In total, 23 SNPs were identified: 14 SNPs on the A-genome and 9 on the C-genome. This indicated that the A-genome (from B. rapa) carried more QTLs for clubroot resistance, but the C-genome (from B. oleracea) could be a potential source for clubroot resistance improvement [13].

One of the major factors that may affect the accuracy of GWAS analysis is the existence of population structure within the population used for GWAS. The analysis confirmed that the core collection of accessions represented two different populations. A multi-locus mixed linear model (MMLM) was used to analysis the association between the phenotypes and the SNP markers because it provided the best fit in Q-Q plots between SNP markers and the DSI for the four pathotypes for the models assessed.

QTLs for clubroot resistance have been identified previously in B. napus [21,22] and several have been mapped to chromosomes $\mathrm{C03}, \mathrm{C06}$, and $\mathrm{C09}$ [13]. We believe that all 23 of the QTLs identified in the current study are novel because they were located at different physical locations on the chromosomes from QTLs identified previously and were associated with resistance to different pathotypes.

The majority of plant disease resistance genes identified to date have been classified as toll-interleukin-1 receptor/nucleotide binding site/leucine-rich repeat (TIR-NBS-LRR or TNL) proteins or coiled coil /nucleotide binding site/leucine-rich repeat (CC-NBS-LRR or CNL) proteins. The ratio of TNLs to CNLs differs among plant species, likely because their $R$ genes are adapted to different pathogens $[23,24]$. About $70 \%$ of NBS-LRR genes in Brassicaceae family belongs to TNLs $[25,26,27]$.

In the current study, 61 resistance genes were identified within $2 \mathrm{Mb}$ upstream and $2 \mathrm{Mb}$ downstream of the SNPs associated with resistance to the four pathotypes. The resistance genes belonged mainly to the TNL family (24 genes) or the CNL family (20 genes). The frequency of TNLs and CNLs was highest on A09 (11 genes) followed in decreasing order by A03, A08, C09, C01 and C03. The uneven distribution of TNLs and CNLs is not uncommon in other plant species [30,31,32,33]. The vast majority of TNLs and CNLs appeared in clusters of 2 to $10 \mathrm{TNLs}$ and CNLs, which is similar to the results of previous studies in B. napus [29], Arabidopsis, Medicago truncatula and Solanum tuberosum [30,34,35]. 
The remaining resistance genes were non-TNL genes (nTNL), comprised of four enhanced disease resistance-like genes, two disease resistance-responsive (dirigent-like protein) family genes, and seven putative disease resistance proteins. A set of nTNLs with RPP13 domain (called RNLs) was also detected. A group of nTNL genes with RPW8 domain (RNL) had been identified in previous studies $[27,36,37]$, but was not observed in the current study.

A previous phylogenetic analysis of nTNLs and CNLs from five Brassicaceae species indicated that RNLs are likely derived from the CNL lineage $[27,29]$. The function of RNLs is yet to be determined, but they have no direct response to the pathogen and may have not the same duplication rates as TNLs and CNLs, which explains their lower abundance in the genome $[27,29]$. They may have a role in defencesignal transduction [38] or as helpers of other NBS genes [38]. The role of other nTNLs is also unknown.

\section{Conclusion}

The current study identified several accessions of $B$. napus with high levels of resistance to four pathotypes of $P$. brassicae. Genome-wide association mapping analysis detected and mapped 23 SNP loci associated with resistance to the four pathotypes. This information will be used in subsequent genetic analysis of bi-parental populations to verify the SNPs and fine map the functional genes responsible for resistance to each pathotype and for marker-assisted breeding of resistance to clubroot in canola.

\section{Materials and methods}

\section{Plant and pathogen materials}

Germplasm of Brassica napus consisting of 177 accessions from 32 countries, provided by three gene banks (Plant Genetic Resources of Canada (PGRC), Centre for Genetic Resources of the Netherlands and Agricultural Research Service, USDA, USA), was selected for study (Table S1). These accessions represented collections from Europe (123 accessions), Asia (29), North America (20), Oceania (2), South America (1), Africa (1), and one accession of unknown origin (Table S1). The accessions were oilseed rape (146 accessions), fodder rapa (21), Swede rape (7), rutabaga (2) and turnip (1). The growth habit was predominantly winter type (129), with some spring type (48) accessions (Table S1).

Plants for GBS analysis were grown in a growth chamber up to the 3-4 leaf stage. A total of $100 \mathrm{mg}$ of leaf tissue was collected from each accession, immediately frozen in liquid nitrogen and then lyophilized in a freeze dryer for approximately $48 \mathrm{~h}$. The freeze-dried tissues were ground to a fine powder using a tissue lyser (Qiagen, Newtown City, USA).

Resting spores of field collections of strains L-G02, F.183-14, F.3-14 and F.1-14 representing pathotypes 5X, 2B, 3A and 3D respectively of $P$. brassicae (Canadian Clubroot Differential) system, [39] were 
increased on canola and stored as frozen clubbed roots at $-20^{\circ} \mathrm{C}$ until needed. Resting spores were extracted from the frozen clubs as described by [40], and adjusted to a concentration of $1 \times 10^{7}$ resting spores $/ \mathrm{mL}$. Spores of each pathotype were applied separately to the host entries.

\section{Evaluation of clubroot reaction}

Seed of each host genotype was pre-germinated on moistened filter paper in a Petri dishes. One-week-old seedlings of each host line and pathotype were inoculated by dipping the entire root system in the resting spore suspension for $10 \mathrm{~s}$. The inoculated seedlings were then immediately planted in $6 \times 6 \times 6 \mathrm{~cm}$ plastic pots filled with Sunshine LA4 potting mixture, with one seedling per pot. The pots were thoroughly watered and transferred to a greenhouse at $21^{\circ} \mathrm{C} \pm 2^{\circ} \mathrm{C}$ with a $16 \mathrm{~h}$ photoperiod. The potting mixture was kept saturated with tap water at $\mathrm{pH} 6.5$ for the first week after inoculation and then watered and fertilized as required.

Six weeks after inoculation, the seedlings were gently removed from the potting mix, the roots of each plant were washed with tap water, and each root was rated for clubroot symptom development on a 0 to 3 scale [41], where: $0=$ no clubs, $1=$ a few small clubs on less than one-third of the roots, $2=$ moderate clubs (small to medium-sized clubs on $1 / 3$ to $2 / 3$ of the roots), and $3=$ severe clubs (medium to largesized clubs on $>2 / 3$ of the roots). A DSI was then calculated using the formula of [42] as modified by [41]:

DSI $\%=\Sigma(n \times 0+n \times 1+n \times 2+n \times 3) N \times 3 \times 100$

Where $n$ is the number of plants in a class; $N$ is the total number of plants in an experimental unit; and 0 , 1,2 and 3 are the symptom severity classes.

\section{Sequence analysis and SNP discovery}

The accession sequences were analyzed using GBS. In brief, GBS involves four major steps: DNA sample preparation, library construction, library sequencing and SNP calling. DNA extraction was performed using the DNeasy 96 plant kit as per the manufacturer's instruction (Qiagen). To reduce the genome complexity, DNA was digested with ApeKI, a methylation-sensitive restriction enzyme. The fragments produced by digestion were directly ligated to enzyme-specific adapters followed by PCR amplification. The samples divided into two pools of 96 samples each followed by two runs of Illumina HiSeq 2500 (Illumina Inc., USA). DNA alignment was generated with BWA software version 0.7.8-r455. The GBSTASSEL pipeline [43] was used for SNP calling, and VCF and HapMap genotype files were generated. Initial SNP filtration was performed with the following settings: MAF > 0.01 and missing data per site < $90 \%$. Accessions with too much missing data were removed. Depth, missingness and heterozygosity were calculated using VCFtools V.0.1.12 [44]. Genotyping and SNP calling was performed the Genomic Diversity Facility, Cornell University (http://www.bio-tech. cornell.edu/brc/brc/ services). 


\section{Variant annotation}

Variants were annotated to regions of the $B$. napus reference genome using $\mathrm{R}$, implemented using "VariantAnnotation" [45], and Variant Effect Predictor (VEP, [46]), and variant locations were characterised as coding, intron, splice site, promoter and intergenic regions.

\section{Genetic diversity and population structure}

Population-based genetic diversity, including allele frequencies, MAF, and average heterozygosity, were computed using TASSEL 5.2.18 software [47]. Polymorphic information content (PIC) values [48] was calculated for SNP markers using the formula $\left.\left(P I C=1-\left(\operatorname{maf}^{\wedge} 2+(1-m a f) \wedge 2\right)\right)-\left(2 m a f^{\wedge} 2(1-m a f)^{\wedge} 2\right)\right)$. The ratio of transitions to transversions was calculated using the [49] 2-parameter model, implemented in MEGA7 [50].

Structure analysis of the accessions was conducted using STRUCTURE software v2.2 [51]. A subset of 10,094 SNPs was selected that was evenly distributed across the genome with one SNP per $100 \mathrm{~Kb}$. The admixture model and correlated allele frequency were applied with a burn-in period of 50,000 iterations and 100,000 replications of Markov Chain Monte Carlo (MCMC). Five runs were performed to calculate the mean likelihood for the number of populations $K$, ranging from 1 to 10 , and the mean of the loglikelihood estimates $\operatorname{LnP}(\mathrm{D})$ for each $\mathrm{K}$. The ad-hoc statistic $\Delta \mathrm{K}$ was used to determine optimal number groups [52]. Structure output was visualized using STRUCTURE HARVESTER web-based software [53]).

\section{Analysis of molecular variance}

Analysis of molecular variance (AMOVA) was conducted using Arlequin v.3.5 software [54] to estimate the genetic variance among clusters and sub-clusters of the $\mathrm{A}$ and $\mathrm{C}$ genome haplotypes. In this analysis, the distance matrix among samples was computed to estimate the genetic structure of the haplotypes. Genetic variance components were estimated, and the total variance was partitioned among major clusters, among sub-clusters within major clusters, and within subclusters. The significance of the variance components was tested using 1,000 permutations. The fixation index $\left(F_{\text {st }}\right)$, an estimation of population differentiation and genetic distance based on genetic polymorphism data, was calculated.

\section{Linkage disequilibrium (LD) analysis}

LD decay across the $B$. napus genome was measured and a correlation matrix of $r^{2}$ values was computed between all pairs of polymorphic SNPs with MAF $\geq 5 \%$ using the GAPIT V2 package [55].

\section{Association analysis}


Data for the disease DSI were transformed using rank-based inverse normal transformation implemented as the rntransform function in the GenABEL R [56]. Association was analyzed for a subset of 10,094 SNP markers with $M A F \geq 5 \%$ using the following models: general linear model (GLM), mixed linear model (MLM), compressed mixed linear model (CMLM), enriched compressed mixed linear model (ECMLM), and multi-locus mixed model (MLMM) implemented in the GAPIT V2 package of R [55]. A kinship matrix of the accessions was calculated and principle components analysis was used to account for population structure and accessions relatedness.

\section{Candidate resistance genes}

Using Blast2Go software [57], the sequence region neighboring (2 Mb upstream and downstream) of the significant SNPs were searched for candidate genes encoding disease resistance proteins potentially responsible for resistance to each pathotype of $P$. brassicae.

\section{List of abbreviations}

AMOVA: analysis of molecular variance; CC: coiled-coil; CCD: Canadian clubroot differential; CMLM: compressed mixed linear model; CNL: CC-NBS-LRR; CR: clubroot resistance; DSI: disease severity index; ECMLM: enriched compressed mixed linear model; GLM: general linear model; GWAS: genome wide association asnalysis; GBS: genotyping by sequencing; LD: linkage disequilibrium; LRR: leucine-rich repeat; MAF: minor allele frequency; MCMC: markov chain monte carlo; MLM: mixed linear model; MMLM: multilocus mixed linear model

NBS: nucleotide-binding site; nTNL: non-TIR-NBS-LRR; PGRC: plant genetic resource of Canada; PIC: Polymorphic information content; Q-Q: quantile-quantile; QTL: quantitative trait locus; SNP: single nucleotide polymorphismTIR: Toll-interleukin-1 receptor; TNL: TIR-NBS-LRR; USDA: United States department of Agriculture; UTR: untranslated region

\section{Acknowledgments}

The authors are grateful to Melissa Kehler, Victor Manolii, Md Mizanur Rahaman and the summer students Yasmina Bekkaoui and Kurtis Flavel for their technical support.

\section{Declarations}

\section{Ethics approval and consent to participate}

Not applicable 


\section{Consent to publish}

Not applicable

\section{Availability of data and materials}

The datasets used and/or analyzed during the current study available from the corresponding author on reasonable request.

\section{Competing interests}

The authors declare that they have no competing interest.

\section{Funding}

This work was funded by a competitive grant from SaskCanola under Canola Agronomic Research Program. The funding body played no role in the design of the study and collection, analysis, and interpretation of data and in writing the manuscript.

\section{Author contributions}

$F Y$ and $A D$ conceived of and designed the study; $A D$ and JW conducted the experiments; $A D, M L, M M K$ and QC analyzed data; SES, SFH, BDG and GP provided important resources and facilities. AD drafted the manuscript. All authors reviewed the manuscript and approved the final draft.

\section{Reference}

- Nagaharu U. Genome analysis in Brassica with special reference to the experimental formation of B. napus and peculiar mode of fertilization. Japanese Journal of Botany. 1935; 7: 389-452.

- Allender CJ, King GJ. Origins of the amphiploid species Brassica napus L. investigated by chloroplast and nuclear molecular markers. BMC Plant Biol. 2010; 10:54. doi:10.1186/1471-222910-54.

- Howell EC, Kearsey MJ, Jones GH, King GJ, Armstrong SJ. A and C genome distinction and chromosome identification in Brassica napus by sequential fluorescence in situ hybridization and genomic in situ hybridization. Genetics. 2008;180(4):1849-1857. doi:10.1534/genetics.108.095893

- Parkin, IAP, and Lydiate, DJ. Conserved patterns of chromosome pairing and recombination in Brassica napus crosses.Genome. 1997; 40: 496-504. 
- McNaughton IH. Swedes and rapes-Brassica napus (Cruciferae). In: Smartt J, Simmonds NW (eds) Evolution of crop plants, 2nd edn. Longman Scientific \& Technical, London, UK. 1995; 68-75.

- Dixon, G. R. The occurrence and economic impact of Plasmodiophora brassicae and clubroot disease. J. Plant Growth Regul. 2009; 28:194-202.

- Karling JS. The Plasmodiophorales 2nd ed. Hafner Publishing Company, Inc., New York (1968).

- Voorrips, RE.Plasmodiophora brassicae: Aspects of pathogenesis and resistance in Brassica oleracea. Euphytica. 1995; 83:139 -146 (1995).

- Diederichsen E, Frauen M, Linders E, Hatakeyama K, Hirai M. Status and perspectives of clubroot resistance breeding in crucifer crops. J. Plant Growth Regul. 2009; 28:265-281.

- Buczacki ST, Toxopeus H, Mattusch P, Johnston TD, Dixon GR, Hobolth LA. Study of physiologic specialization in Plasmodiophora brassicae: proposals for attempted rationalization through an international approach. Transaction of British Mycological Society. 1975; 65:295-303.

- Piao Z, Ramchiary N, Lim YP. Genetics of clubroot resistance in Brassica species. J. Plant Growth Regul. 2009; 28: 252-264.

- Rahman H, Shakir A, Hasan MJ. Breeding for clubroot resistant spring canola (Brassica napus L.) for the Canadian prairies: Can the European winter canola cv. Mendel be used as a source of resistance? Can. J. Plant Sci. 2011; 91: 447-458.

- Li L, Luo Y, Chen B, et al. A Genome-Wide Association Study Reveals New Loci for Resistance to Clubroot Disease in Brassica napus. Front Plant Sci. 2016;7:1483. doi:10.3389/fpls.2016.01483

- Flint-Garcia SA, Thuillet AC, Yu J, Pressoir G, Romero SM, et al. Maize association population: a highresolution platform for quantitative trait locus dissection. Plant J. 2005; 44: 1054-1064

- Gupta PK, Rustgi S, Kulwal PL. Linkage disequilibrium and association studies in higher plants: present status and future prospects. Plant Mol. Biol. 2005; 57: 461-485.

- Elshire RJ, Glaubitz JC, Sun Q, et al. A robust, simple genotyping-by-sequencing (GBS) approach for high diversity species. PLoS One. 2011; 6:e19379. doi:10.1371/journal.pone.0019379.

- Crossa J, Burgueno J, Dreisigacker S, et al. Association analysis of historical bread wheat germplasm using additive genetic covariance of relatives and population structure. Genetics. 2007; 177:1889-1913.

- Ranc N, Muños S, Xu J, et al. Genome-wide association mapping in tomato (Solanum lycopersicum) is possible using genome admixture of Solanum lycopersicum var. cerasiforme. G3. 2012; 2: 853864. doi:10.1534/g3.112.002667.

- Kump KL, Bradbury PJ, Wisser RJ, Buckler ES, Belcher AR, et al. Genome-wide association study of quantitative resistance to southern leaf blight in the maize nested association mapping population. Nat. Genet. 2011; 43: 163-168.

- Wang N, Chen B, Xu K, et al. Association Mapping of Flowering Time QTLs and Insight into Their Contributions to Rapeseed Growth Habits. Front Plant Sci. 2016;7:338. doi:10.3389/fpls.2016.00338. 
- Manzanares-Dauleux MJ, Delourme R, Baron F, Thomas G. Mapping of one major gene and of QTLs involved in resistance to clubroot in Brassica napus. Theor. Appl. Genet. 2000; 101: 885-891. $10.1007 /$ s001220051557

- Werner S, Diederichsen E, Frauen M, Schondelmaier J, Jung C. Genetic mapping of clubroot resistance genes in oilseed rape. Theor. Appl. Genet. 2008; 116: 363-372. 10.1007/s00122-0070674-2.

- Leister D. Tandem and segmental gene duplication and recombination in the evolution of plant disease resistance genes. Trends In Genetics: TIG. 2004; 20: 116-122. doi:10.1016/j.tig.2004.01.007.

- Lozano R, Ponce O, Ramirez M, Mostajo N, Orjeda G. Genome-wide identification and mapping of NBS-encoding resistance genes in Solanum tuberosum Group Phureja. PLoS One. 2012; 7: e34775. doi:10.1371/ journal.pone.0034775

- Mun J, Yu H, Park S, Park B. Genome-wide identification of NBS encoding resistance genes in Brassica rapa. Molecular Genetics and Genomics. 2009; 282: 617-631. doi:10.1007/s00438-0090492-0.

- Yu J,Tehrim S, Zhang F,Tong C, Huang J, Cheng X, Dong C, Zhou Y, Qin R, Hua W, Liu S. Genome-wide comparative analysis of NBSencoding genes between Brassica species and Arabidopsis thaliana. BMC Genomics. 2014; 15: 3. doi:10.1186/1471-2164-15-3.

- Zhang YM, Shao ZQ, Wang Q, Hang YY, Xue JY, Wang B, Chen JQ. Uncovering the dynamic evolution of nucleotide-binding site-leucinerich repeat (NBS-LRR) genes in Brassicaceae. Journal of Integrative Plant Biology. 2016; 58:165-177. doi:10.1111/jipb.12365.

- Dakouri A, Zhang X, Peng G et al. Analysis of genome-wide variants through bulked segregant RNA sequencing reveals a major gene for resistance to Plasmodiophora brassicae in Brassica oleracea. Scientific Reports. 2018; 8:17657.

- Alamery S, Tirnaz S, Bayer P, et al. Genome-wide identification and comparative analysis of NBS-LRR resistance genes in Brassica napus. Crop Pasture Sci. 2017; 69: 79-94. doi: 10.1071/CP17214.

- Meyers B, Kozik A, Griego A, Kuang H, Michelmore R. Genome-wide analysis of NBS-LRR-encoding genes in Arabidopsis. The Plant Cell. 2003; 15: 809-834. doi:10.1105/tpc.009308.

- Zhou T, Wang Y, Chen JQ, Araki H, Jing Z, Jiang K, Shen J, Tian D. Genome-wide identification of NBS genes in japonica rice reveals significant expansion of divergent non-TIR NBS-LRR genes. Molecular Genetics and Genomics. 2004; 271: 402-415. doi:10.1007/s00438004-0990-z.

- Kohler A, Rinaldi C, Duplessis S, Baucher M, Geelen D, Duchaussoy F, Meyers B, Boerjan W, Martin F. Genome-wide identification of NBS resistance genes in Populus trichocarpa. Plant Molecular Biology. 2008; 66: 619-636. doi:10.1007/s11103-008-9293-9.

- Yang S, Zhang X, Yue J-X, Tian D, Chen J-Q. Recent duplications dominate NBS-encoding gene expansion in two woody species. Molecular Genetics and Genomics. 2008; 280: 187-198. doi:10.1007/s00438008-0355-0. 
- Ameline-Torregrosa C, Wang B-B, O'Bleness MS, Deshpande S, Zhu H, Roe B, Young ND, Cannon SB. Identification and characterization of nucleotide-binding site-leucine-rich repeat genes in the model plant Medicago truncatula. Plant Physiology. 2008; 146: 5-21. doi:10.1104/pp.107.104588.

- Jupe F, Pritchard L, Etherington G, MacKenzie K, Cock P, Wright F, Sharma SK, Bolser D, Bryan G, Jones J, Hein I. Identification and localisation of the NB-LRR gene family within the potato genome. BMC Genomics. 2012; 13: 75. doi:10.1186/1471-2164-13-75.

- Bonardi V, Tang S, Stallmann A, Roberts M, Cherkis K, Dangl JL. Expanded functions for a family of plant intracellular immune receptors beyond specific recognition of pathogen effectors. Proceedings of the National Academy of Sciences of the United States of America. 2011; 108: 16463-16468. doi:10.1073/pnas.1113726108

- Collier SM, Hamel L-P, Moffett P. Cell death mediated by the N-terminal domains of a unique and highly conserved class of NB-LRR protein. Molecular Plant-Microbe Interactions. 2011; 24: 918-931. doi:10.1094/MPMI-03-11-0050.

- Shao Z-Q, Zhang Y-M, Hang Y-Y, Xue J-Y, Zhou G-C, Wu P, Wu X-Y, Wu X-Z, Wang Q, Wang B. Longterm evolution of nucleotide binding site-leucine-rich repeat genes: understanding gained from and beyond the legume family. Plant Physiology. 2014; 166: 217-234. doi:10.1104/pp.114.243626.

- Strelkov, S. E., Hwang, S. F., Manolii, V. P., Cao, T., Fredua-Agyeman, R., Harding, M. W., Peng, G., Gossen, B. D., McDonald, M. R., and Feindel, D. Virulence and pathotype classification of Plasmodiophora brassicae populations collected from clubroot resistant canola (Brassica napus) in Canada. Can. J. Plant Pathol. 20185; 40: 284-298. DOI: 10.1080/07060661.2018.1459851.

- Strelkov SE, Tewari JP, Smith-Degenhardt E. Characterization of Plasmodiophora brassicaepopulations from Alberta, Canada. Canadian Journal of Plant Pathology. 2006; 28: 467474.

- Kuginuki Y, Yoshikawa H, Hirai M. Eur. J. Plant Pathol. 1999; 105:327-332.

- Horiuchi S, and Hori M. Bull. Chugoku Natl. Agric. Exp. Stn. Ser. E. (Environ. Div.), 1980; 17:33-55.

- Glaubitz JC., Casstevens TM, Lu F, Harriman J, Elshire RJ, Sun Q, Buckler ES. TASSEL-GBS: A High Capacity Genotyping by Sequencing Analysis Pipeline. PLoS ONE. 2014; 9:E90346. doi:10.1371/journal.pone.0090346

- Danecek P, Auton A, Abecasis G, et al. The variant call format and VCFtools. Bioinformatics. 2011; 27:2156-2158. doi:10.1093/bioinformatics/btr330

- Obenchain V, Lawrence M, Carey V, Gogarten S, Shannon P and Morgan M. "VariantAnnotation: a Bioconductor package for exploration and annotation of genetic variants." Bioinformatics. 2014; 30 : 2076-2078. doi: 10.1093/bioinformatics/btu168.

- McLaren W, Gil L, Hunt SE, Riat HS, Ritchie GR, Thormann A, Flicek P, Cunningham F.The Ensembl Variant Effect Predictor. Genome Biology 2016; 17:122. doi:10.1186/s13059-016-0974-4.

- Bradbury PJ, Zhang Z, Kroon DE, Casstevens TM, Ramdoss Y, Buckler ES.TASSEL: Software for association mapping of complex traits in diverse samples. Bioinformatics. 2007; 23: 2633-2635. doi:10.1093/bioinformatics/btm308. PMID:17586829. 
- Roussel V, Koenig J, Beckert M, Balfourier F. Molecular diversity in French bread wheat accessions related to temporal trends and breeding programmes. Theor Appl Genet. 2004; 108:920-930

- Kimura M. A simple method for estimating evolutionary rate of base substitutions through comparative studies of nucleotide sequences. Journal of Molecular Evolution. 1980; 16:111-120.

- Kumar S, Stecher G, Tamura K. MEGA7: molecular evolutionary genetics analysis version 7.0 for bigger datasets. Mol Biol Evol. 2016; 33:1870-1874.

- Pritchard JK, Stephens M, Donnelly P. Inference of population structure using multilocus genotype data. Genetics. 2000; 155: 945-959.

- Evanno G, Regnaut S, Goudet J. Detecting the number of clusters of individuals using the software structure: a simulation study. Mol. Ecol. 2005; 4: 2611-2620. doi:10.1111/j.1365-294X.2005.02553.

- Earl DA, VonHoldt BM. STRUCTURE HARVESTER: a website and program for visualizing STRUCTURE output and implementing the Evanno method. Conserv Genet Resour. 2011; 4: 359-361.

- Excoffier L, Lischer HEL. Arlequin suite ver 3.5: A new series of programs to perform population genetics analyses under Linux and Windows. Mol Ecol Resour. 2010; 10: 564-567.

- Tang Y, Liu X, Wang J, Li M, Wang Q, Tian F, et al. GAPIT Version 2: An enhanced integrated tool for genomic association and prediction. Plant Genome. 2018; 9: 2-9. doi: 10.3835/plantgenome2015.11.0120.

- Aulchenko YS, Ripke S, Isaacs A, Van Duijn CM.. GenABEL: an R library for genome-wide association analysis. Bioinformatics. 2007; 23: 1294-1296.

- Götz S, García-Gómez JM, Terol J, Williams TD, Nagaraj SH, Nueda MJ, Conesa, A. High-throughput functional annotation and data mining with the Blast2GO suite. Nucleic acids research. $2008 ; 36$ : 3420-3435. doi:10.1093/nar/gkn176.

Table 1 Genome wide distribution of SNPs, minor allele frequency (MAF), Heterozygosity, polymorphic information content (PIC) and average Linkage disequilibrium (LD)

Chromosome

Start

End

Total No. seq

SNP

$\mathrm{SNP} / \mathrm{Kb}$

MAF

Heterozygosity 
PIC

Average LD

A01

2024

23251220

23250

13062

1.78

0.14

0.08

0.24

0.090

A02

919

24785167

24784

12455

1.99

0.13

0.08

0.23

0.080

A03

808

Page 20/73 
29746073

29745

20541

1.45

0.14

0.07

0.24

0.060

A04

1717

19141470

19140

10562

1.81

0.14

0.07

0.24

0.090

A05

2697

23052978

23050

14917

1.55

Page 21/73 
0.14

0.06

0.24

0.076

A06

2120

24372251

24370

14696

1.66

0.13

0.06

0.22

0.075

A07

10938

24000655

23990

14232

1.69

0.15

0.07

0.24

0.070

Page 22/73 
A08

1729

18958296

18957

10281

1.84

0.13

0.07

0.22

0.084

A09

1327

33857792

33857

18702

1.81

0.12

0.07

0.21

0.010

A10

4083

17366872

17363

Page 23/73 
12131

1.43

0.14

0.07

0.23

0.080

Average (A-subgenome)

23851

14158

1.70

0.14

0.07

0.23

0.072

C01

8039

38812658

38805

17087

2.27

0.16

0.08

0.27

0.190 
C02

1607

46186975

46185

17662

2.61

0.14

0.09

0.24

0.146

C03

760

60565276

60565

25136

2.41

0.15

0.09

0.24

0.073

Chromosome

Start

End

Total No. seq 
SNP

$\mathrm{SNP} / \mathrm{Kb}$

MAF

Heterozygosity

PIC

Average LD

C04

1773

48929072

48927

19053

2.57

0.14

0.08

0.24

0.140

C05

3386

43172068

43169

16540

2.61

0.13

0.10

Page 26/73 
0.22

0.074

C06

1745

37224854

37223

14761

2.52

0.14

0.09

0.23

0.079

C07

7046

44766293

44760

15558

2.88

0.13

0.09

0.22

0.083

C08

6385

Page 27/73 
38472912

38467

16082

2.39

0.14

0.09

0.23

0.105

C09

1884

48501448

48500

18295

2.65

0.12

0.09

0.21

0.075

Average (C-subgenome)

45178

17797

2.55

0.14

0.09 
0.23

0.107

Average (genome)

34514

15978

2.12

0.14

0.08

0.23

0.088

Table 2 List of significant SNPs, chromosomes, physical location and P values

Pathotype

SNP locus

Chromosome

Position

P.value

$-\log (P$ values $)$

A01_19406286

A01

19406286

$2.49 \mathrm{E}-07$

6.60

$5 X$

A08_171171159

Page 29/73 
A08

2719211

5.39E-06

5.27

A09_195497763

A09

8083774

1.11E-05

4.96

C08_579178095

C08

20763231

4.88E-05

4.31

A07_147954943

A07

3509616

$9.95 \mathrm{E}-10$

9.00

A04_83864566

A04

6035183

1.15E-07

6.94

Page $30 / 73$ 
A03_651104541

A03

1393088

5.23E-07

6.28

A08_186203638

A08

17751690

$3.94 \mathrm{E}-06$

5.40

2B

C01_276968702

C01

38290946

5.56E-06

5.26

C03_688995474

$\mathrm{CO3}$

6293335

7.75E-06

5.11

A05_115772286

A05

18791143

Page 31/73 
$9.44 \mathrm{E}-06$

5.02

A09_215839211

A09

28425222

2.18E-05

4.66

A03_68863700

A03

20801907

3.63E-05

4.44

C01_341096049

C01

17366972

$1.95 \mathrm{E}-08$

7.71

A10_222415846

A10

1136417

$5.56 \mathrm{E}-08$

7.25

$3 \mathrm{~A}$

C04_403341747

Page $32 / 73$ 
C04

19039176

3.50E-07

6.46

C09_608174205

C09

11282154

$6.73 \mathrm{E}-07$

6.17

C09_634081519

C09

37189468

2.21E-05

4.66

A04_90743077

A04

12913694

7.21E-07

6.14

C09_638459286

C09

41567235

2.76E-06

5.56

Page 33/73 
A03_71057307

A03

22995514

3.13E-06

5.50

A01_17862282

A01

17862282

3.31E-06

5.48

C03_685453245

$\mathrm{CO3}$

2751106

4.46E-05

4.35

Table 3 List of disease resistance genes located within candidate gene region (Mb) of SNP loci

Pathotype

SNP

Chromosome

Gene ID

Candidate gene region $(\mathrm{Mb})$

Distance to SNP (Mb)

Description 
$5 X$

A01_19406286

A01

BnaA01g28560D

$19-20$

0.47

Disease resistance

A03

BnaA03g03110D

$1-2$

0.11

ENHANCED Disease RESISTANCE 2-like

2B

A03_651104541

A03

BnaA03g03260D

$1-2$

0.19

TIR-NBS-LRR class gene

A03

BnaA03g03270D

$1-2$

0.20

TIR-NBS-LRR class gene 
$\mathrm{A} 03$

BnaA03g43880D

$20.5-23.5$

1.30

ENHANCED Disease RESISTANCE 2-like isoform X1

$\mathrm{A} 03$

BnaA03g44070D

$20.5-23.5$

0.73

TIR-NBS-LRR class gene

A03

BnaA03g44080D

$20.5-23.5$

0.73

TIR-NBS-LRR class gene

$\mathrm{A} 03$

BnaA03g44090D

$20.5-23.5$

0.73

Disease resistance RRS1-like isoform X1

2B

A03_68863700

$\mathrm{A} 03$

BnaA03g45000D 
$20.5-23.5$

0.13

TIR-NBS-LRR class gene

A03

BnaA03g45010D

$20.5-23.5$

0.12

TIR-NBS-LRR class gene

A03

BnaA03g45020D

$20.5-23.5$

0.12

TIR-NBS-LRR class gene

3D

A03_71057307

A03

BnaA03g45040D

$20.5-23.5$

0.11

TIR-NBS-LRR class gene

A03

BnaA03g45050D

$20.5-23.5$

0.11 
TIR-NBS-LRR class gene

A03

BnaA03g45970D

$20.5-23.5$

0.46

TIR-NBS-LRR class gene

A04

BnaA04g06520D

$5.5-6.5$

0.92

Putative Disease resistance protein

A04

BnaA04g06530D

$5.5-6.5$

0.92

Putative Disease resistance protein

2B

A04_83864566

A04

BnaA04g06550D

$5.5-6.5$

0.91

Putative Disease resistance protein A04 
BnaA04g06580D

$5.5-6.5$

0.82

Putative Disease resistance protein

A04

BnaA04g06780D

$5.5-6.5$

0.63

Disease resistance-responsive (dirigent-like protein)

2B

A05_115772286

A05

BnaA05g24990D

$18-19$

0.22

CC-NBS-LRR class gene

A05

BnaA05g25000D

$18-19$

0.21

CC-NBS-LRR class gene

$5 X$

A08_171171159

A08 
BnaA08g02210D

$1-3$

0.94

CC-NBS-LRR class gene

A08

BnaA08g24820D

$17-18$

0.52

CC-NBS-LRR class gene

A08

BnaA08g24860D

$17-18$

0.49

CC-NBS-LRR class gene

$2 B$

A08_186203638

A08

BnaA08g26370D

$17-18$

0.12

CC-NBS-LRR class gene

A08

BnaA08g26380D

$17-18$ 
0.13

CC-NBS-LRR class gene

A08

BnaA08g26400D

17-18

0.14

CC-NBS-LRR class gene

A08_186203638

A08

BnaA08g26410D

$17-18$

0.14

CC-NBS-LRR class gene

A09

BnaA09g13280D

7-9

0.76

TIR-NBS-LRR class gene

A09

BnaA09g13850D

7-9

0.20

TIR-NBS-LRR class gene A09 
BnaA09g13890D

7-9

0.16

TIR-NBS-LRR class gene

A09

BnaA09g13900D

7-9

0.15

TIR-NBS-LRR class gene

$5 X$

A09_195497763

A09

BnaA09g14320D

7-9

0.11

TIR-NBS-LRR class gene

A09

BnaA09g14420D

7-9

8.26

CC-NBS-LRR class gene

A09

BnaA09g14550D

7-9 
0.30

CC-NBS-LRR class gene

A09

BnaA09g14560D

7-9

0.30

CC-NBS-LRR class gene A09

BnaA09g14570D

7-9

0.31

CC-NBS-LRR class gene A09

BnaA09g14680D

7-9

0.37

CC-NBS-LRR class gene A09

BnaA09g42680D

$27.1-30.1$

1.28

CC-NBS-LRR class gene

A09

BnaA09g43420D 
$27.1-30.1$

1.66

Disease resistance-responsive (dirigent-like protein) family

2B

A09_215839211

A09

BnaA09g43430D

$27.1-30.1$

1.67

Disease resistance-responsive (dirigent-like protein) family A09

BnaA09g39390D

$27.1-30.1$

0.51

ENHANCED Disease RESISTANCE 4-like

A09

BnaA09g39400D

$27.1-30.1$

0.51

ENHANCED Disease RESISTANCE 4-like

A10

BnaA10g04000D

$1-3$

0.98 
ENHANCED DISEASE RESISTANCE-like protein (DUF1336)

3A

A10_222415846

A10

BnaA10g05000D

$1-3$

1.60

CC-NBS-LRR class gene

$\mathrm{C} 01$

BnaC01g39050D

$37-39$

0.46

Disease resistance protein RPS6 isoform X1

$\mathrm{C} 01$

BnaC01g40270D

$37-39$

0.36

TIR-NBS-LRR class gene

$3 \mathrm{~A}$

C01

BnaC01g40280D

37-39

0.36

TIR-NBS-LRR class gene 
C01_276968702

C01

BnaC01g40300D

$37-39$

0.37

TIR-NBS-LRR class gene

C01

BnaC01g40310D

$37-39$

0.38

TIR-NBS-LRR class gene

C01

BnaC01g40460D

$37-39$

0.44

Putative Disease resistance protein At4g11170

2B

C03_685453245

$\mathrm{CO3}$

BnaC03g04690D

$2-3$

0.48

TIR-NBS-LRR class gene

3D 
C03_688995474

$\mathrm{CO3}$

BnaC03g05380D

$2-3$

0.16

TIR-NBS-LRR class gene

3A

C04_403341747

C04

BnaC04g18730D

17-19

0.48

CC-NBS-LRR class gene

$5 X$

C08_579178095

C08

BnaC08g17450D

20.7-21.1

0.31

TIR-NBS-LRR class gene

C09

BnaC09g14400D

$11-12$

0.26 
TIR-NBS-LRR class gene

C09

BnaC09g14870D

$11-12$

0.12

TIR-NBS-LRR class gene

$3 \mathrm{~A}$

C09_608174205

C09

BnaC09g15010D

$11-12$

0.24

CC-NBS-LRR class gene

C09

BnaC09g15020D

$11-12$

0.24

CC-NBS-LRR class gene

C09

BnaC09g15110D

$11-12$

0.39

CC-NBS-LRR class gene

$3 D$ 
A09_638459286

C09

BnaC09g38250D

$41-42$

0.38

Disease resistance

\section{Figure legends}

Fig 1. Frequency distribution of accessions plotted against clubroot severity (disease severity index, DSI) for four pathotypes $5 \mathrm{X}, 2 \mathrm{~B}, 3 \mathrm{~A}$, and $3 \mathrm{D}$ indicated in the figure.

Fig 2. Population structure analysis of the 177 accessions based on $A$. model-based Bayesian clustering using STRUCTURE for $\mathrm{K}=2$ groups, and $B$. estimation of the number of sub-populations for $\mathrm{K}$ values of 1 to 10 .

Fig 3. Manhattan plots of association analysis using the multilocus mixed linear model (MMLM) model $\mathrm{P}+\mathrm{K}$ for pathotypes $A$. 5X, B. 2B, C. 3A and D. 3D. The horizontal line represents the threshold of significance $\left(-\log _{10} 0.5 / 10094=4.30\right)$.

\section{Additional files}

Table S1 List of accessions, their growth habits, type, origin and DSIs.

Table S2 Pearson correlation coefficient $\left(\mathrm{r}^{2}\right)$ between DSIs of the four pathotypes.

Table S3 Analysis of molecular variance (AMOVA) design and results.

Table S4 Distance method: Sub-Cluster Pairwise FST differences.

Figure S1 Frequency distribution of rank-transformed disease severity index for pathotypes (a) 5X-LG2, (b) 2B, (c) 3A, and (d) 3D.

Figure S2 Relative frequency distribution (\%) of Bi-/Multi-allelic SNPs

Figure S3 Variant annotation results, $A$. the distribution of SNPs within genic regions; coding region, introns and promotor region, $B$. Derailed SNP annotation based on Variant Effect Predictor software, $C$. Distribution of annotated SNP across B. napus chromosomes. 
Figure S4 Phylogenetic analysis of the 177 B. napus accessions based SNP markers $A$. Major cluster A and subclusters SCA-I, SCA-II and SCA-III, B. Major cluster B and subcluster SCB-I, SCB-II, SCB-III. The geographical distribution at the continent level is also illustrated.

Figure S5 Genome wide linkage disequilibrium (LD) decay plot as a function of physical distance (bp). LD decay assessed in a $B$. napus collection of 177 accessions LD estimates are reported as squared correlations of allele frequencies (r2).

\section{Results}

\section{Evaluation of clubroot reaction}

At 6 weeks after seeding, the germplasm was evaluated for resistance to four $P$. brassicae pathotypes; $5 \mathrm{X}$, $2 \mathrm{~B}, 3 \mathrm{~A}$ and $3 \mathrm{D}$. The disease severity index (DSI) ranged from 0 to 100 . The majority of accessions were highly to completely susceptible (70-100 DSI), but several were highly resistant (0-20 DSI) to pathotypes 5X (21 accessions), 2B (7 accessions), 3A (8 accessions), and 3D (15 accessions) (Fig.1, Table S1). The correlation coefficient of severity among the four pathotypes was strongest between $2 \mathrm{~B}$ and $3 \mathrm{~A}\left(r^{2}=\right.$ $0.77)$ and weakest between $5 X$ and $3 D\left(r^{2}=0.27\right.$, Table S2). Phenotypic data were transformed using rank-based inverse normal transformation to make the DSI values nearly fit the normal distribution required for parametric model-based association analysis (Figure S1).

\section{Sequence analysis and SNP discovery}

Genotyping by sequencing (GBS) data analysis was performed for the 177 B. napus accessions. A total of $\sim 1.2$ billion reads and $\sim 633$ million good barcoded reads were generated and split into three FASTQ fills. On average, there were $3.3 \mathrm{M}$ read counts per sample (range $\sim 1.8$ to $7.7 \mathrm{M}$ ) and $3.1 \mathrm{M}$ read counts mapped (range 76 to $96 \%$ ). Sequence tags from each file were captured and merged to produce a master tag file of $4,253,499$ sequence tags. The tags were then aligned to $B$. napus reference genome v4.1, using the TASSEL-GBS pipeline. A total of 2,217,292 (52.1\%) tags were uniquely aligned to the reference, $1,220,090(28.7 \%)$ aligned to multiple positions and 816,117 (19.2\%) were not aligned. Uniquely mapped tags were used to calculate the tag density distribution at each site in the $B$. napus genome and for SNP calling.

The raw sequence data for SNP calling were also analysed using the TASSEL-GBS pipeline. A total of 399,234 unfiltered SNPs and 355,680 filtered SNPs were called for the 177 accessions, with a mean of individual depth of $8.5 \pm 2$ SD and mean site depth of $6.7 \pm 11.4$ SD. Of the 355,680 filtered SNPs, 301,753 SNPs were mapped to the 19 chromosomes; the remaining SNPs were randomly distributed without specific chromosome assignment. Only variants mapped to chromosomes were kept for further analyses. 


\section{Variant analysis and annotation}

There were more SNPs in the C-genome (160,174 SNPs) than the A-genome (141,579 SNPs).

Chromosome A03 had the highest number of SNPs within the A-genome, while $\mathrm{C03}$ contained the highest number of SNPs in the C-genome (Table 1). The mean density per Kb was 2.12 SNP / Kb across the 19 chromosomes. In general, SNP density was higher in the C- genome (2.55 SNPs / Kb) than the A-genome (1.70 SNPs / Kb). C07 had the highest number of SNPs per Kb (2.88) and A10 had the lowest (1.43) (Table 1). The vast majority of SNPs were bi-allelic (90\%), and only $10 \%$ were multi-allelic (Figure S2). There was a positive correlation $\left(r^{2}=0.80\right)$ between chromosome length and the number of SNPs, but only a weak correlation $\left(r^{2}=0.3\right)$ between the number of SNPs and the number of SNPs per Kb.

The SNPs were annotated using the VariantAnnotation package of R. About $37 \%$ of SNPs were annotated within coding regions, $22 \%$ within introns, $31 \%$ within promoter regions, $0.3 \%$ within splice sites, and $9.7 \%$ mapped to other genetic regions (Figure S3). A more detailed SNP annotation was performed using the Variant Effect Predictor (Figure S3). For SNPs within coding regions, 17\% were non-synonymous, 18\% were upstream-gene variants, $9 \%$ were downstream-gene variants, $23 \%$ were synonymous variants, $14 \%$ were intron variants, $15 \%$ intergenic variants, and $4 \%$ were located in the splice site regions and $5^{\prime}$ and $3^{\prime}$ UTRs (Figure S2C). Overall, more SNPs were annotated to the A-genome than the C-genome (Figure S3).

\section{Genetic diversity and population structure}

For genetic diversity analysis, the SNP markers were filtered at a minor allele frequency (MAF) of 0.05 and minimum sample count of $80 \%$, which resulted in 140,195 good quality SNPs. The mean MAF was the same for the $\mathrm{A}$ - and $\mathrm{C}$-genomes (MAF $=0.14)$. Chromosome $\mathrm{C} 01$ had the highest MAF $(0.16)$, followed by $\mathrm{C} 03$ and A07 (0.15), and lowest in chromosomes A09 and C09 (0.12) (Table 1). The mean marker heterozygosity $\left(\mathrm{H}_{\mathrm{e}}\right)$ was 0.06 and the mean accession heterozygosity was 0.14 . The average polymorphic information content (PIC) was the same for A and C-genomes (0.26). PIC was highest in chromosome C01 (0.27) and lowest (0.24) in A09 (Table 1). The ratio of transitions (changes from $A<->$ and $C<->$ )

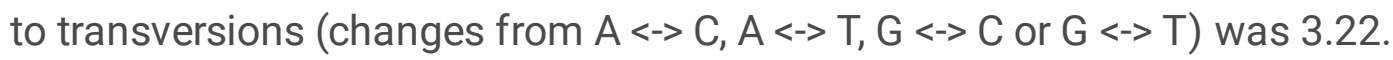

Population structure analysis indicated the existence of two major group populations, and analysis using the Evanno criterion supported this result (Fig. 2). Population 1 contained 63 accessions (35.6\%) representing all continents, while population 2 contained 114 accessions (64.4\%), mainly from Europe. A phylogenetic tree using the neighbour-joining algorithm produced two major clusters and six subclusters (Figure S4).

\section{Analysis of molecular variance}

Analysis of molecular variance on the six subclusters (SCA-I, II, III,SCB-I, II and III) identified significant genetic differences between major clusters, among subclusters, and among individuals within sub- 
clusters $(p<0.001)$. Variance within subclusters accounted for $87.7 \%$ of the total variance, with only $7.5 \%$ among sub-cluster and $4.7 \%$ among major clusters. The fixation index $\left(F_{s t}\right)$ value was 0.21 , which indicated that the accessions belonged to two closely related groups (Table S3). Sub-cluster pairwise $F_{\text {st }}$ values ranged from 0.03 between SCB-I and SCB-II to 0.16 between SCA-I and SCB-II (Table S4).

\section{Linkage disequilibrium analysis}

Linkage disequilibrium in the association panel was calculated using Pearson's $r^{2}$ statistic on pairwise combinations of SNPs present across the 19 chromosomes of B. napus (Figure S5). The average LD ( $\left.r^{2}\right)$ across the genome was 0.15 . The mean LD was 0.10 in the A-genome and 0.19 in the C-genome. LD values ranged from 0.01 in $\mathrm{A} 09$ to 0.19 in $\mathrm{C} 01$ (Table 1). Across the genome, LD decayed very rapidly $\left(r^{2}=\right.$ 0.20 ) within $300 \mathrm{~Kb}$ (Figure S5).

\section{Association analysis}

Genome-wide association analysis for clubroot severity was conducted using the following models: general linear model (GLM), mixed linear model (MLM), compressed mixed linear model (CMLM), enriched compressed mixed linear model (ECMLM), and multi-locus mixed model (MLMM). The quantilequantile (Q-Q) plots, from all models revealed that, save for significant SNPs, the distribution of observed $-\log 10(p)$ was closest to the expected distribution in the MLMM compared to other models, therefore associations were identified using this model. A significance threshold of $P<0.5 / \mathrm{N}$ (N: number of SNPs) was used for detecting significant SNPs. The MLMM-genome-wide association study (GWAS) detected 23 SNPs associated with resistance to the four $P$. brassicae pathotypes including four SNPs associated with resistance to $5 \mathrm{X}$, nine SNPs to $2 \mathrm{~B}$, five to $3 \mathrm{~A}$ and five to $3 \mathrm{D}$. The name, physical position, $P$ value and $-\log (P$ value $)$ are presented in Table 2. Across genome, the A-genome carried 14 SNP loci and the Cgenome carried 11 loci (Table 2, Fig. 3).

\section{Candidate resistance genes}

A Blast search identified 61 nucleotide binding site/leucine-rich repeat (NBS-LRR) resistance proteins and non-NBS-LRR resistance genes within the $2 \mathrm{Mb}$ sequence upstream and downstream of 19 out of 23 significant SNP loci detected in our study (Table 3). The majority of resistance genes appeared as clusters of 2 to 10 genes, while they appeared as a single gene in other cases. On A01, one resistance gene (BnaA01g28560D) was found at $\sim 0.5 \mathrm{Mb}$ from the A01_19406286 locus associated with resistance to pathotype 5X. On A03, one Enhanced Disease Resistance 2-like (BnaA03g03110D) gene and two TIRNBS-LRR resistance (BnaA03g03260D, BnaA03g03270D) genes were detected at 0.11-0.2 Mb distance from A03_651104541 locus associated with resistance to pathotype 2B. Additionally, a cluster of two TIRNBS-LRR resistance (BnaA03g44070D, BnaA03g44080D) genes and a Disease Resistance RRS1-like isoform X1 were detected at $0.73 \mathrm{Mb}$ distance from A03_68863700 locus associated with resistance to 
pathotype 2B (Table 3). A cluster of six TIR-NBS-LRR resistance (BnaA03g45000D, BnaA03g45010D, $B n a A 03 g 45020 D, B n a A 03 g 45040 D, B n a A 03 g 45050 D)$ genes were identified on A03 at 0.11-0.13 Mb from the A03_71057307 locus associated with resistance to pathotype 3D (Table 3). On A04, a gene (BnaA04g06780D) encoding a Disease Resistance-Responsive (dirigent-like protein) protein family and four putative disease resistance genes (BnaA04g06520D, BnaA04g06530D, BnaA04g06550D, BnaA04g06580D) were identified at 0.63-0.92 Mb from A04_83864566 locus associated with resistance to 2B. On A05, two CC-NBS-LRR (BnaA05g24990D, BnaA05g25000D) genes were identified at $\sim 0.2 \mathrm{Mb}$ from A05_115772286 locus associated with resistance to pathotype 2B. On A08, one disease resistance gene (BnaA08g02210D) was detected at $0.94 \mathrm{Mb}$ from A08_171171159 locus associated with resistance to pathotype $5 \mathrm{X}$. Also, a cluster of six CC-NBS-LRR genes were found at $0.12-0.52 \mathrm{Mb}$ from A08_186203638 associated with resistance to 2B. On A09, a cluster of five TIR-NBS-LRR resistance genes (BnaA09g13280D, BnaA09g13850D, BnaA09g13890D, BnaA09g13900D, BnaA09g14320D) and five CCNBS-LRR genes (BnaA09g14420D, BnaA09g14550D, BnaA09g14560D, BnaA09g14570D, BnaA09g14580D) were detected at 0.11-0.76 Mb from A09_195497763 locus associated with resistance to 5X. In addition, one CC-NBS-LRR gene (BnaA09g42680D),, two Disease Resistance-Responsive genes (dirigent-like protein) and two Enhanced Disease Resistance 4-like genes were found at 0.50-1.67 Mb from A09_215839211 locus associated with resistance to pathotype 2B. On A10, one enhanced disease resistance-like gene (BnaA10g04000D), , and one CC-NBS-LRR gene (BnaA10g05000D) were detected at 0.98-1.50 Mb from the A10_222415846 locus associated with resistance to pathotype 3A.

On C01, four TIR-NBS-LRR resistance genes (BnaC01g40270D, BnaC01g40280D, BnaC01g40300D, BnaC01g40310D) and two non-NBS-LRR disease resistance genes (BnaC01g39050D, BnaC01g40460D) were identified at $0.36-0.46 \mathrm{Mb}$ from the locus at C01_276968702 associated with resistance to pathotype 3A (Table 3). On C03, two TIR-NBS-LRR resistance genes (BnaC03g05380D, BnaC03g04690D) located at $0.16 \mathrm{Mb}$ and $0.48 \mathrm{Mb}$ from C03_68899547 and C03_685453245 loci associated with resistance to pathotype 3D were detected. On C04, one TIR-NBS-LRR resistance gene (BnaC08g17450D) and one CC-NBS-LRR resistance gene (BnaC04g18730D) mapped at $0.31 \mathrm{Mb}$ and $0.48 \mathrm{Mb}$, respectively, from the C04_403341747 locus associated with resistance to pathotype 3A (Table 3). On C08, one TIRNBS-LRR resistance (BnaC08g17450D) gene was identified at $0.31 \mathrm{Mb}$ from C08_579178095 locus associated with resistance to 5X. On C09, two TIR-NBS-LRR resistance genes (BnaC09g 14400D, BnaC09g14870D) and three CC-NBS-LRR resistance genes (BnaC09g15010D, BnaC09g15020D, BnaC09g15110D) located at 0.12-0.4 Mb from C09_608174205 locus associated with resistance to $3 \mathrm{~A}$. In addition, a non-NBS-LRR disease resistance gene (BnaC09g38250D) located at $0.38 \mathrm{Mb}$ from C09_638459286 locus associated with resistance to 3D was detected (Table 3).

\section{Discussion}

GWAS has been widely used to identify and map QTLs for quantitatively inherited traits in a wide range of plant species. In the current study, GWAS was used to identify and map new sources of resistance to four highly aggressive pathotypes (5X,2B, 3A, 3D) of $P$. brassicae in 177 accessions of $B$. napus. The majority of the accessions were highly susceptible to all four pathotypes (80-100 DSI), while 10\% 
showed high levels of resistance (0-25 DSI). This supported previous reports that sources of high levels of resistance to clubroot were much less common in B. napus than in B. rapa [11,12]. In total, 23 SNPs were identified: 14 SNPs on the A-genome and 9 on the C-genome. This indicated that the A-genome (from B. rapa) carried more QTLs for clubroot resistance, but the C-genome (from B. oleracea) could be a potential source for clubroot resistance improvement [13].

One of the major factors that may affect the accuracy of GWAS analysis is the existence of population structure within the population used for GWAS. The analysis confirmed that the core collection of accessions represented two different populations. A multi-locus mixed linear model (MMLM) was used to analysis the association between the phenotypes and the SNP markers because it provided the best fit in Q-Q plots between SNP markers and the DSI for the four pathotypes for the models assessed.

QTLs for clubroot resistance have been identified previously in $B$. napus $[21,22]$ and several have been mapped to chromosomes $\mathrm{C03}, \mathrm{C06}$, and $\mathrm{C09}$ [13]. We believe that all 23 of the QTLs identified in the current study are novel because they were located at different physical locations on the chromosomes from QTLs identified previously and were associated with resistance to different pathotypes.

The majority of plant disease resistance genes identified to date have been classified as toll-interleukin-1 receptor/nucleotide binding site/leucine-rich repeat (TIR-NBS-LRR or TNL) proteins or coiled coil /nucleotide binding site/leucine-rich repeat (CC-NBS-LRR or CNL) proteins. The ratio of TNLs to CNLs differs among plant species, likely because their $\mathrm{R}$ genes are adapted to different pathogens [23,24]. About $70 \%$ of NBS-LRR genes in Brassicaceae family belongs to TNLs $[25,26,27]$.

In the current study, 61 resistance genes were identified within $2 \mathrm{Mb}$ upstream and $2 \mathrm{Mb}$ downstream of the SNPs associated with resistance to the four pathotypes. The resistance genes belonged mainly to the TNL family (24 genes) or the CNL family (20 genes). The frequency of TNLs and CNLs was highest on A09 (11 genes) followed in decreasing order by A03, A08, C09, $\mathrm{C} 01$ and $\mathrm{C} 03$. The uneven distribution of TNLs and CNLs is not uncommon in other plant species [30,31,32,33]. The vast majority of TNLs and CNLs appeared in clusters of 2 to 10 TNLs and CNLs, which is similar to the results of previous studies in B. napus [29], Arabidopsis, Medicago truncatula and Solanum tuberosum [30,34,35].

The remaining resistance genes were non-TNL genes (nTNL), comprised of four enhanced disease resistance-like genes, two disease resistance-responsive (dirigent-like protein) family genes, and seven putative disease resistance proteins. A set of nTNLs with RPP13 domain (called RNLs) was also detected. A group of nTNL genes with RPW8 domain (RNL) had been identified in previous studies $[27,36,37]$, but was not observed in the current study.

A previous phylogenetic analysis of nTNLs and CNLs from five Brassicaceae species indicated that RNLs are likely derived from the CNL lineage $[27,29]$. The function of RNLs is yet to be determined, but they have no direct response to the pathogen and may have not the same duplication rates as TNLs and CNLs, which explains their lower abundance in the genome $[27,29]$. They may have a role in defencesignal transduction [38] or as helpers of other NBS genes [38]. The role of other nTNLs is also unknown. 


\section{Conclusion}

The current study identified several accessions of $B$. napus with high levels of resistance to four pathotypes of $P$. brassicae. Genome-wide association mapping analysis detected and mapped 23 SNP loci associated with resistance to the four pathotypes. This information will be used in subsequent genetic analysis of bi-parental populations to verify the SNPs and fine map the functional genes responsible for resistance to each pathotype and for marker-assisted breeding of resistance to clubroot in canola.

\section{Materials And Methods \\ Plant and pathogen materials}

Germplasm of Brassica napus consisting of 177 accessions from 32 countries, provided by three gene banks (Plant Genetic Resources of Canada (PGRC), Centre for Genetic Resources of the Netherlands and Agricultural Research Service, USDA, USA), was selected for study (Table S1). These accessions represented collections from Europe (123 accessions), Asia (29), North America (20), Oceania (2), South America (1), Africa (1), and one accession of unknown origin (Table S1). The accessions were oilseed rape (146 accessions), fodder rapa (21), Swede rape (7), rutabaga (2) and turnip (1). The growth habit was predominantly winter type (129), with some spring type (48) accessions (Table S1).

Plants for GBS analysis were grown in a growth chamber up to the 3-4 leaf stage. A total of $100 \mathrm{mg}$ of leaf tissue was collected from each accession, immediately frozen in liquid nitrogen and then lyophilized in a freeze dryer for approximately $48 \mathrm{~h}$. The freeze-dried tissues were ground to a fine powder using a tissue lyser (Qiagen, Newtown City, USA).

Resting spores of field collections of strains L-G02, F.183-14, F.3-14 and F.1-14 representing pathotypes 5X, 2B, 3A and 3D respectively of $P$. brassicae (Canadian Clubroot Differential) system, [39] were increased on canola and stored as frozen clubbed roots at $-20^{\circ} \mathrm{C}$ until needed. Resting spores were extracted from the frozen clubs as described by [40], and adjusted to a concentration of $1 \times 10^{7}$ resting spores $/ \mathrm{mL}$. Spores of each pathotype were applied separately to the host entries.

\section{Evaluation of clubroot reaction}

Seed of each host genotype was pre-germinated on moistened filter paper in a Petri dishes. One-week-old seedlings of each host line and pathotype were inoculated by dipping the entire root system in the resting spore suspension for $10 \mathrm{~s}$. The inoculated seedlings were then immediately planted in $6 \times 6 \times 6 \mathrm{~cm}$ plastic pots filled with Sunshine LA4 potting mixture, with one seedling per pot. The pots were thoroughly watered and transferred to a greenhouse at $21^{\circ} \mathrm{C} \pm 2{ }^{\circ} \mathrm{C}$ with a $16 \mathrm{~h}$ photoperiod. The potting mixture was kept saturated with tap water at $\mathrm{pH} 6.5$ for the first week after inoculation and then watered and fertilized as required. 
Six weeks after inoculation, the seedlings were gently removed from the potting mix, the roots of each plant were washed with tap water, and each root was rated for clubroot symptom development on a 0 to 3 scale [41], where: $0=$ no clubs, $1=$ a few small clubs on less than one-third of the roots, $2=$ moderate clubs (small to medium-sized clubs on $1 / 3$ to $2 / 3$ of the roots), and $3=$ severe clubs (medium to largesized clubs on $>2 / 3$ of the roots). A DSI was then calculated using the formula of [42] as modified by [41]:

[Due to technical limitations, this equation is only available as a download in the supplemental files section.]

Where $n$ is the number of plants in a class; $N$ is the total number of plants in an experimental unit; and 0 , 1,2 and 3 are the symptom severity classes.

\section{Sequence analysis and SNP discovery}

The accession sequences were analyzed using GBS. In brief, GBS involves four major steps: DNA sample preparation, library construction, library sequencing and SNP calling. DNA extraction was performed using the DNeasy 96 plant kit as per the manufacturer's instruction (Qiagen). To reduce the genome complexity, DNA was digested with ApeKI, a methylation-sensitive restriction enzyme. The fragments produced by digestion were directly ligated to enzyme-specific adapters followed by PCR amplification. The samples divided into two pools of 96 samples each followed by two runs of Illumina HiSeq 2500 (IIlumina Inc., USA). DNA alignment was generated with BWA software version 0.7.8-r455. The GBSTASSEL pipeline [43] was used for SNP calling, and VCF and HapMap genotype files were generated. Initial SNP filtration was performed with the following settings: MAF > 0.01 and missing data per site < $90 \%$. Accessions with too much missing data were removed. Depth, missingness and heterozygosity were calculated using VCFtools V.0.1.12 [44]. Genotyping and SNP calling was performed the Genomic Diversity Facility, Cornell University (http://www.bio-tech. cornell.edu/brc /brc/ services).

\section{Variant annotation}

Variants were annotated to regions of the $B$. napus reference genome using $\mathrm{R}$, implemented using "VariantAnnotation" [45], and Variant Effect Predictor (VEP, [46]), and variant locations were characterised as coding, intron, splice site, promoter and intergenic regions.

\section{Genetic diversity and population structure}

Population-based genetic diversity, including allele frequencies, MAF, and average heterozygosity, were computed using TASSEL 5.2.18 software [47]. Polymorphic information content (PIC) values [48] was calculated for SNP markers using the formula $\left.\left(P I C=1-\left(m a f^{\wedge} 2+(1-m a f) \wedge 2\right)\right)-\left(2 m a f^{\wedge} 2(1-m a f)^{\wedge} 2\right)\right)$. The 
ratio of transitions to transversions was calculated using the [49] 2-parameter model, implemented in MEGA7 [50].

Structure analysis of the accessions was conducted using STRUCTURE software v2.2 [51]. A subset of 10,094 SNPs was selected that was evenly distributed across the genome with one SNP per $100 \mathrm{~Kb}$. The admixture model and correlated allele frequency were applied with a burn-in period of 50,000 iterations and 100,000 replications of Markov Chain Monte Carlo (MCMC). Five runs were performed to calculate the mean likelihood for the number of populations $K$, ranging from 1 to 10 , and the mean of the loglikelihood estimates $\mathrm{LnP}(\mathrm{D})$ for each $\mathrm{K}$. The ad-hoc statistic $\Delta \mathrm{K}$ was used to determine optimal number groups [52]. Structure output was visualized using STRUCTURE HARVESTER web-based software [53]).

\section{Analysis of molecular variance}

Analysis of molecular variance (AMOVA) was conducted using Arlequin v.3.5 software [54] to estimate the genetic variance among clusters and sub-clusters of the $A$ and $C$ genome haplotypes. In this analysis, the distance matrix among samples was computed to estimate the genetic structure of the haplotypes. Genetic variance components were estimated, and the total variance was partitioned among major clusters, among sub-clusters within major clusters, and within subclusters. The significance of the variance components was tested using 1,000 permutations. The fixation index $\left(F_{s t}\right)$, an estimation of population differentiation and genetic distance based on genetic polymorphism data, was calculated.

\section{Linkage disequilibrium (LD) analysis}

LD decay across the $B$. napus genome was measured and a correlation matrix of $r^{2}$ values was computed between all pairs of polymorphic SNPs with MAF $\geq 5 \%$ using the GAPIT V2 package [55].

\section{Association analysis}

Data for the disease DSI were transformed using rank-based inverse normal transformation implemented as the rntransform function in the GenABEL R [56]. Association was analyzed for a subset of 10,094 SNP markers with $M A F \geq 5 \%$ using the following models: general linear model (GLM), mixed linear model (MLM), compressed mixed linear model (CMLM), enriched compressed mixed linear model (ECMLM), and multi-locus mixed model (MLMM) implemented in the GAPIT V2 package of R [55]. A kinship matrix of the accessions was calculated and principle components analysis was used to account for population structure and accessions relatedness.

\section{Candidate resistance genes}


Using Blast2Go software [57], the sequence region neighboring (2 Mb upstream and downstream) of the significant SNPs were searched for candidate genes encoding disease resistance proteins potentially responsible for resistance to each pathotype of $P$. brassicae.

\section{List Of Abbreviations}

AMOVA: analysis of molecular variance; CC: coiled-coil; CCD: Canadian clubroot differential; CMLM: compressed mixed linear model; CNL: CC-NBS-LRR; CR: clubroot resistance; DSI: disease severity index; ECMLM: enriched compressed mixed linear model; GLM: general linear model; GWAS: genome wide association asnalysis; GBS: genotyping by sequencing; LD: linkage disequilibrium; LRR: leucine-rich repeat; MAF: minor allele frequency; MCMC: markov chain monte carlo; MLM: mixed linear model; MMLM: multilocus mixed linear model; NBS: nucleotide-binding site; nTNL: non-TIR-NBS-LRR; PGRC: plant genetic resource of Canada; PIC: Polymorphic information content; Q-Q: quantile-quantile; QTL: quantitative trait locus; SNP: single nucleotide polymorphismTIR: Toll-interleukin-1 receptor; TNL: TIRNBS-LRR; USDA: United States department of Agriculture; UTR: untranslated region

\section{Declarations}

\section{Acknowledgments}

The authors are grateful to Melissa Kehler, Victor Manolii, Md Mizanur Rahaman and the summer students Yasmina Bekkaoui and Kurtis Flavel for their technical support.

\section{Ethics approval and consent to participate}

Not applicable

\section{Consent to publish}

Not applicable

\section{Availability of data and materials}

The datasets used and/or analyzed during the current study available from the corresponding author on reasonable request.

\section{Competing interests}

The authors declare that they have no competing interest. 


\section{Funding}

This work was funded by a competitive grant from SaskCanola under Canola Agronomic Research Program. The funding body played no role in the design of the study and collection, analysis, and interpretation of data and in writing the manuscript.

\section{Author contributions}

$F Y$ and $A D$ conceived of and designed the study; $A D$ and JW conducted the experiments; $A D, M L, M M K$ and QC analyzed data; SES, SFH, BDG and GP provided important resources and facilities. AD drafted the manuscript. All authors reviewed the manuscript and approved the final draft.

\section{Reference}

1. Nagaharu U. Genome analysis in Brassica with special reference to the experimental formation of B. napus and peculiar mode of fertilization. Japanese Journal of Botany. 1935; 7: 389-452.

2. Allender CJ, King GJ. Origins of the amphiploid species Brassica napus L. investigated by chloroplast and nuclear molecular markers. BMC Plant Biol. 2010; 10:54. doi:10.1186/1471-222910-54.

3. Howell EC, Kearsey MJ, Jones GH, King GJ, Armstrong SJ. A and C genome distinction and chromosome identification in Brassica napus by sequential fluorescence in situ hybridization and genomic in situ hybridization. Genetics. 2008;180(4):1849-1857. doi:10.1534/genetics.108.095893

4. Parkin, IAP, and Lydiate, DJ. Conserved patterns of chromosome pairing and recombination in Brassica napus crosses. Genome. 1997; 40: 496-504.

5. McNaughton IH. Swedes and rapes-Brassica napus (Cruciferae). In: Smartt J, Simmonds NW (eds) Evolution of crop plants, 2nd edn. Longman Scientific \& Technical, London, UK. 1995; 68-75.

6. Dixon, G. R. The occurrence and economic impact of Plasmodiophora brassicae and clubroot disease. J. Plant Growth Regul. 2009; 28:194-202.

7. Karling JS. The Plasmodiophorales 2nd ed. Hafner Publishing Company, Inc., New York (1968).

8. Voorrips, RE.Plasmodiophora brassicae: Aspects of pathogenesis and resistance in Brassica oleracea. Euphytica. 1995; 83:139 -146 (1995).

9. Diederichsen E, Frauen M, Linders E, Hatakeyama K, Hirai M. Status and perspectives of clubroot resistance breeding in crucifer crops. J. Plant Growth Regul. 2009; 28:265-281.

10. Buczacki ST, Toxopeus H, Mattusch P, Johnston TD, Dixon GR, Hobolth LA. Study of physiologic specialization in Plasmodiophora brassicae: proposals for attempted rationalization through an international approach. Transaction of British Mycological Society. 1975; 65:295-303.

11. Piao Z, Ramchiary N, Lim YP. Genetics of clubroot resistance in Brassica species. J. Plant Growth Regul. 2009; 28: 252-264. 
12. Rahman H, Shakir A, Hasan MJ. Breeding for clubroot resistant spring canola (Brassica napus L.) for the Canadian prairies: Can the European winter canola cv. Mendel be used as a source of resistance? Can. J. Plant Sci. 2011; 91: 447-458.

13. Li L, Luo Y, Chen B, et al. A Genome-Wide Association Study Reveals New Loci for Resistance to Clubroot Disease in Brassica napus. Front Plant Sci. 2016;7:1483. doi:10.3389/fpls.2016.01483

14. Flint-Garcia SA, Thuillet AC, Yu J, Pressoir G, Romero SM, et al. Maize association population: a highresolution platform for quantitative trait locus dissection. Plant J. 2005; 44: 1054-1064

15. Gupta PK, Rustgi S, Kulwal PL. Linkage disequilibrium and association studies in higher plants: present status and future prospects. Plant Mol. Biol. 2005; 57: 461-485.

16. Elshire RJ, Glaubitz JC, Sun Q, et al. A robust, simple genotyping-by-sequencing (GBS) approach for high diversity species. PLoS One. 2011; 6:e19379. doi:10.1371/journal.pone.0019379.

17. Crossa J, Burgueno J, Dreisigacker S, et al. Association analysis of historical bread wheat germplasm using additive genetic covariance of relatives and population structure. Genetics. 2007; 177:1889-1913.

18. Ranc N, Muños S, Xu J, et al. Genome-wide association mapping in tomato (Solanum lycopersicum) is possible using genome admixture of Solanum lycopersicum var. cerasiforme. G3. 2012; 2: 853864. doi:10.1534/g3.112.002667.

19. Kump KL, Bradbury PJ, Wisser RJ, Buckler ES, Belcher AR, et al. Genome-wide association study of quantitative resistance to southern leaf blight in the maize nested association mapping population. Nat. Genet. 2011; 43: 163-168.

20. Wang N, Chen B, Xu K, et al. Association Mapping of Flowering Time QTLs and Insight into Their Contributions to Rapeseed Growth Habits. Front Plant Sci. 2016;7:338. doi:10.3389/fpls.2016.00338.

21. Manzanares-Dauleux MJ, Delourme R, Baron F, Thomas G. Mapping of one major gene and of QTLs involved in resistance to clubroot in Brassica napus. Theor. Appl. Genet. 2000; 101: 885-891. $10.1007 /$ s001220051557

22. Werner S, Diederichsen E, Frauen M, Schondelmaier J, Jung C. Genetic mapping of clubroot resistance genes in oilseed rape. Theor. Appl. Genet. 2008; 116: 363-372. 10.1007/s00122-0070674-2.

23. Leister $D$. Tandem and segmental gene duplication and recombination in the evolution of plant disease resistance genes. Trends In Genetics: TIG. 2004; 20: 116-122. doi:10.1016/j.tig.2004.01.007.

24. Lozano R, Ponce O, Ramirez M, Mostajo N, Orjeda G. Genome-wide identification and mapping of NBS-encoding resistance genes in Solanum tuberosum Group Phureja. PLoS One. 2012; 7: e34775. doi:10.1371/ journal.pone.0034775

25. Mun J, Yu H, Park S, Park B. Genome-wide identification of NBS encoding resistance genes in Brassica rapa. Molecular Genetics and Genomics. 2009; 282: 617-631. doi:10.1007/s00438-0090492-0. 
26. Yu J,Tehrim S, Zhang F,Tong C, Huang J, Cheng X, Dong C, Zhou Y, Qin R, Hua W, Liu S. Genome-wide comparative analysis of NBSencoding genes between Brassica species and Arabidopsis thaliana. BMC Genomics. 2014; 15: 3. doi:10.1186/1471-2164-15-3.

27. Zhang YM, Shao ZQ, Wang Q, Hang YY, Xue JY, Wang B, Chen JQ. Uncovering the dynamic evolution of nucleotide-binding site-leucinerich repeat (NBS-LRR) genes in Brassicaceae. Journal of Integrative Plant Biology. 2016; 58:165-177. doi:10.1111/jipb.12365.

28. Dakouri A, Zhang X, Peng G et al. Analysis of genome-wide variants through bulked segregant RNA sequencing reveals a major gene for resistance to Plasmodiophora brassicae in Brassica oleracea. Scientific Reports. 2018; 8:17657.

29. Alamery S, Tirnaz S, Bayer $P$, et al. Genome-wide identification and comparative analysis of NBS-LRR resistance genes in Brassica napus. Crop Pasture Sci. 2017; 69: 79-94. doi: 10.1071/CP17214.

30. Meyers B, Kozik A, Griego A, Kuang H, Michelmore R. Genome-wide analysis of NBS-LRR-encoding genes in Arabidopsis. The Plant Cell. 2003; 15: 809-834. doi:10.1105/tpc.009308.

31. Zhou T, Wang Y, Chen JQ, Araki H, Jing Z, Jiang K, Shen J, Tian D. Genome-wide identification of NBS genes in japonica rice reveals significant expansion of divergent non-TIR NBS-LRR genes. Molecular Genetics and Genomics. 2004; 271: 402-415. doi:10.1007/s00438004-0990-z.

32. Kohler A, Rinaldi C, Duplessis S, Baucher M, Geelen D, Duchaussoy F, Meyers B, Boerjan W, Martin F. Genome-wide identification of NBS resistance genes in Populus trichocarpa. Plant Molecular Biology. 2008; 66: 619-636. doi:10.1007/s11103-008-9293-9.

33. Yang S, Zhang X, Yue J-X, Tian D, Chen J-Q. Recent duplications dominate NBS-encoding gene expansion in two woody species. Molecular Genetics and Genomics. 2008; 280: 187-198. doi:10.1007/s00438008-0355-0.

34. Ameline-Torregrosa C, Wang B-B, O'Bleness MS, Deshpande S, Zhu H, Roe B, Young ND, Cannon SB. Identification and characterization of nucleotide-binding site-leucine-rich repeat genes in the model plant Medicago truncatula. Plant Physiology. 2008; 146: 5-21. doi:10.1104/pp.107.104588.

35. Jupe F, Pritchard L, Etherington G, MacKenzie K, Cock P, Wright F, Sharma SK, Bolser D, Bryan G, Jones J, Hein I. Identification and localisation of the NB-LRR gene family within the potato genome. BMC Genomics. 2012; 13: 75. doi:10.1186/1471-2164-13-75.

36. Bonardi V, Tang S, Stallmann A, Roberts M, Cherkis K, Dangl JL. Expanded functions for a family of plant intracellular immune receptors beyond specific recognition of pathogen effectors. Proceedings of the National Academy of Sciences of the United States of America. 2011; 108: 16463-16468. doi:10.1073/pnas.1113726108

37. Collier SM, Hamel L-P, Moffett P. Cell death mediated by the N-terminal domains of a unique and highly conserved class of NB-LRR protein. Molecular Plant-Microbe Interactions. 2011; 24: 918-931. doi:10.1094/MPMI-03-11-0050.

38. Shao Z-Q, Zhang Y-M, Hang Y-Y, Xue J-Y, Zhou G-C, Wu P, Wu X-Y, Wu X-Z, Wang Q, Wang B. Longterm evolution of nucleotide binding site-leucine-rich repeat genes: understanding gained from and beyond the legume family. Plant Physiology. 2014; 166: 217-234. doi:10.1104/pp.114.243626. 
39. Strelkov, S. E., Hwang, S. F., Manolii, V. P., Cao, T., Fredua-Agyeman, R., Harding, M. W., Peng, G., Gossen, B. D., McDonald, M. R., and Feindel, D. Virulence and pathotype classification of Plasmodiophora brassicae populations collected from clubroot resistant canola (Brassica napus) in Canada. Can. J. Plant Pathol. 20185; 40: 284-298. DOI: 10.1080/07060661.2018.1459851.

40. Strelkov SE, Tewari JP, Smith-Degenhardt E. Characterization of Plasmodiophora brassicaepopulations from Alberta, Canada. Canadian Journal of Plant Pathology. 2006; 28: 467474.

41. Kuginuki Y, Yoshikawa H, Hirai M. Eur. J. Plant Pathol. 1999; 105:327-332.

42. Horiuchi S, and Hori M. Bull. Chugoku Natl. Agric. Exp. Stn. Ser. E. (Environ. Div.), 1980; 17:33-55.

43. Glaubitz JC., Casstevens TM, Lu F, Harriman J, Elshire RJ, Sun Q, Buckler ES. TASSEL-GBS: A High Capacity Genotyping by Sequencing Analysis Pipeline. PLoS ONE. 2014; 9:E90346. doi:10.1371/journal.pone.0090346

44. Danecek P, Auton A, Abecasis G, et al. The variant call format and VCFtools. Bioinformatics. 2011; 27:2156-2158. doi:10.1093/bioinformatics/btr330

45. Obenchain V, Lawrence M, Carey V, Gogarten S, Shannon P and Morgan M. "VariantAnnotation: a Bioconductor package for exploration and annotation of genetic variants." Bioinformatics. 2014; 30 : 2076-2078. doi: 10.1093/bioinformatics/btu168.

46. McLaren W, Gil L, Hunt SE, Riat HS, Ritchie GR, Thormann A, Flicek P, Cunningham F.The Ensembl Variant Effect Predictor. Genome Biology 2016; 17:122. doi:10.1186/s13059-016-0974-4.

47. Bradbury PJ, Zhang Z, Kroon DE, Casstevens TM, Ramdoss Y, Buckler ES.TASSEL: Software for association mapping of complex traits in diverse samples. Bioinformatics. 2007; 23: 2633-2635. doi:10.1093/bioinformatics/btm308. PMID:17586829.

48. Roussel V, Koenig J, Beckert M, Balfourier F. Molecular diversity in French bread wheat accessions related to temporal trends and breeding programmes. Theor Appl Genet. 2004; 108:920-930

49. Kimura M. A simple method for estimating evolutionary rate of base substitutions through comparative studies of nucleotide sequences. Journal of Molecular Evolution. 1980; 16:111-120.

50. Kumar S, Stecher G, Tamura K. MEGA7: molecular evolutionary genetics analysis version 7.0 for bigger datasets. Mol Biol Evol. 2016; 33:1870-1874.

51. Pritchard JK, Stephens M, Donnelly P. Inference of population structure using multilocus genotype data. Genetics. 2000; 155: 945-959.

52. Evanno G, Regnaut S, Goudet J. Detecting the number of clusters of individuals using the software structure: a simulation study. Mol. Ecol. 2005; 4: 2611-2620. doi:10.1111/j.1365-294X.2005.02553.

53. Earl DA, VonHoldt BM. STRUCTURE HARVESTER: a website and program for visualizing STRUCTURE output and implementing the Evanno method. Conserv Genet Resour. 2011; 4: 359-361.

54. Excoffier L, Lischer HEL. Arlequin suite ver 3.5: A new series of programs to perform population genetics analyses under Linux and Windows. Mol Ecol Resour. 2010; 10: 564-567. 
55. Tang Y, Liu X, Wang J, Li M, Wang Q, Tian F, et al. GAPIT Version 2: An enhanced integrated tool for genomic association and prediction. Plant Genome. 2018; 9: 2-9. doi:

10.3835/plantgenome2015.11.0120.

56. Aulchenko YS, Ripke S, Isaacs A, Van Duijn CM.. GenABEL: an R library for genome-wide association analysis. Bioinformatics. 2007; 23: 1294-1296.

57. Götz S, García-Gómez JM, Terol J, Williams TD, Nagaraj SH, Nueda MJ, Conesa, A. High-throughput functional annotation and data mining with the Blast2GO suite. Nucleic acids research. 2008; 36 : 3420-3435. doi:10.1093/nar/gkn176.

\section{Tables}

Table 1 Genome wide distribution of SNPs, minor allele frequency (MAF), Heterozygosity, polymorphic information content (PIC) and average Linkage disequilibrium (LD) 


\begin{tabular}{|c|c|c|c|c|c|c|c|c|c|}
\hline Chromosome & Start & End & $\begin{array}{c}\text { Total } \\
\text { No. } \\
\text { seq }\end{array}$ & SNP & $\mathrm{SNP} / \mathrm{Kb}$ & MAF & Heterozygosity & PIC & $\begin{array}{l}\text { Average } \\
\text { LD }\end{array}$ \\
\hline $\mathrm{A} 01$ & 2024 & 23251220 & 23250 & 13062 & 1.78 & 0.14 & 0.08 & 0.24 & 0.090 \\
\hline A02 & 919 & 24785167 & 24784 & 12455 & 1.99 & 0.13 & 0.08 & 0.23 & 0.080 \\
\hline A03 & 808 & 29746073 & 29745 & 20541 & 1.45 & 0.14 & 0.07 & 0.24 & 0.060 \\
\hline A04 & 1717 & 19141470 & 19140 & 10562 & 1.81 & 0.14 & 0.07 & 0.24 & 0.090 \\
\hline A05 & 2697 & 23052978 & 23050 & 14917 & 1.55 & 0.14 & 0.06 & 0.24 & 0.076 \\
\hline A06 & 2120 & 24372251 & 24370 & 14696 & 1.66 & 0.13 & 0.06 & 0.22 & 0.075 \\
\hline A07 & 10938 & 24000655 & 23990 & 14232 & 1.69 & 0.15 & 0.07 & 0.24 & 0.070 \\
\hline A08 & 1729 & 18958296 & 18957 & 10281 & 1.84 & 0.13 & 0.07 & 0.22 & 0.084 \\
\hline A09 & 1327 & 33857792 & 33857 & 18702 & 1.81 & 0.12 & 0.07 & 0.21 & 0.010 \\
\hline A10 & 4083 & 17366872 & 17363 & 12131 & 1.43 & 0.14 & 0.07 & 0.23 & 0.080 \\
\hline $\begin{array}{l}\text { Average (A- } \\
\text { subgenome) }\end{array}$ & & & 23851 & 14158 & 1.70 & 0.14 & 0.07 & 0.23 & 0.072 \\
\hline $\mathrm{C01}$ & 8039 & 38812658 & 38805 & 17087 & 2.27 & 0.16 & 0.08 & 0.27 & 0.190 \\
\hline $\mathrm{C} 02$ & 1607 & 46186975 & 46185 & 17662 & 2.61 & 0.14 & 0.09 & 0.24 & 0.146 \\
\hline C03 & 760 & 60565276 & 60565 & 25136 & 2.41 & 0.15 & 0.09 & 0.24 & 0.073 \\
\hline Chromosome & Start & End & $\begin{array}{c}\text { Total } \\
\text { No. } \\
\text { seq }\end{array}$ & SNP & $\mathrm{SNP} / \mathrm{Kb}$ & $\overline{\mathrm{MAF}}$ & Heterozygosity & PIC & Average LD \\
\hline C04 & 1773 & 48929072 & 48927 & 19053 & 2.57 & 0.14 & 0.08 & 0.24 & 0.140 \\
\hline $\mathrm{C} 05$ & 3386 & 43172068 & 43169 & 16540 & 2.61 & 0.13 & 0.10 & 0.22 & 0.074 \\
\hline C06 & 1745 & 37224854 & 37223 & 14761 & 2.52 & 0.14 & 0.09 & 0.23 & 0.079 \\
\hline $\mathrm{C} 07$ & 7046 & 44766293 & 44760 & 15558 & 2.88 & 0.13 & 0.09 & 0.22 & 0.083 \\
\hline $\mathrm{C} 08$ & 6385 & 38472912 & 38467 & 16082 & 2.39 & 0.14 & 0.09 & 0.23 & 0.105 \\
\hline C09 & 1884 & 48501448 & 48500 & 18295 & 2.65 & 0.12 & 0.09 & 0.21 & 0.075 \\
\hline $\begin{array}{l}\text { Average (C- } \\
\text { subgenome) }\end{array}$ & & & 45178 & 17797 & 2.55 & 0.14 & 0.09 & 0.23 & 0.107 \\
\hline $\begin{array}{l}\text { Average } \\
\text { (genome) }\end{array}$ & & & 34514 & 15978 & 2.12 & 0.14 & 0.08 & 0.23 & 0.088 \\
\hline
\end{tabular}


Table 2 List of significant SNPs, chromosomes, physical location and P values

\begin{tabular}{|c|c|c|c|c|c|}
\hline Pathotype & SNP locus & Chromosome & Position & P.value & $-\log (P$ values $)$ \\
\hline \multirow{4}{*}{$5 \mathrm{X}$} & A01_19406286 & A01 & 19406286 & $2.49 \mathrm{E}-07$ & 6.60 \\
\hline & A08_171171159 & A08 & 2719211 & 5.39E-06 & 5.27 \\
\hline & A09_195497763 & A09 & 8083774 & $1.11 \mathrm{E}-05$ & 4.96 \\
\hline & C08_579178095 & C08 & 20763231 & 4.88E-05 & 4.31 \\
\hline \multirow{9}{*}{$2 B$} & A07_147954943 & A07 & 3509616 & $9.95 \mathrm{E}-10$ & 9.00 \\
\hline & A04_83864566 & A04 & 6035183 & $1.15 \mathrm{E}-07$ & 6.94 \\
\hline & A03_651104541 & A03 & 1393088 & 5.23E-07 & 6.28 \\
\hline & A08_186203638 & A08 & 17751690 & $3.94 \mathrm{E}-06$ & 5.40 \\
\hline & C01_276968702 & C01 & 38290946 & 5.56E-06 & 5.26 \\
\hline & C03_688995474 & $\mathrm{CO3}$ & 6293335 & 7.75E-06 & 5.11 \\
\hline & A05_115772286 & A05 & 18791143 & $9.44 \mathrm{E}-06$ & 5.02 \\
\hline & A09_215839211 & A09 & 28425222 & 2.18E-05 & 4.66 \\
\hline & A03_68863700 & A03 & 20801907 & 3.63E-05 & 4.44 \\
\hline \multirow{5}{*}{$3 \mathrm{~A}$} & C01_341096049 & $\mathrm{C} 01$ & 17366972 & $1.95 \mathrm{E}-08$ & 7.71 \\
\hline & A10_222415846 & A10 & 1136417 & 5.56E-08 & 7.25 \\
\hline & C04_403341747 & C04 & 19039176 & 3.50E-07 & 6.46 \\
\hline & C09_608174205 & $\mathrm{C} 09$ & 11282154 & $6.73 \mathrm{E}-07$ & 6.17 \\
\hline & C09_634081519 & C09 & 37189468 & $2.21 \mathrm{E}-05$ & 4.66 \\
\hline \multirow{5}{*}{$3 \mathrm{D}$} & A04_90743077 & $\mathrm{A} 04$ & 12913694 & $7.21 \mathrm{E}-07$ & 6.14 \\
\hline & C09_638459286 & $\mathrm{C} 09$ & 41567235 & 2.76E-06 & 5.56 \\
\hline & A03_71057307 & A03 & 22995514 & 3.13E-06 & 5.50 \\
\hline & A01_17862282 & A01 & 17862282 & 3.31E-06 & 5.48 \\
\hline & C03_685453245 & $\mathrm{C03}$ & 2751106 & 4.46E-05 & 4.35 \\
\hline
\end{tabular}


Table 3 List of disease resistance genes located within candidate gene region $(\mathrm{Mb})$ of SNP loci 


\begin{tabular}{|c|c|c|c|c|c|c|}
\hline Pathotype & SNP & Chromosome & Gene ID & $\begin{array}{l}\text { Candidate } \\
\text { gene } \\
\text { region } \\
(\mathrm{Mb})\end{array}$ & $\begin{array}{l}\text { Distance } \\
\text { to SNP } \\
(\mathrm{Mb})\end{array}$ & Description \\
\hline $5 \mathrm{X}$ & A01_19406286 & A01 & BnaA01g28560D & $19-20$ & 0.47 & Disease resistance \\
\hline \multirow{3}{*}{ 2B } & \multirow{3}{*}{ A03_651104541 } & A03 & BnaA03g03110D & $1-2$ & 0.11 & $\begin{array}{l}\text { ENHANCED } \\
\text { Disease }\end{array}$ \\
\hline & & A03 & BnaA03g03260D & $1-2$ & 0.19 & $\begin{array}{l}\text { RESISTANCE 2-like } \\
\text { TIR-NBS-LRR class } \\
\text { gene }\end{array}$ \\
\hline & & A03 & BnaA03g03270D & $1-2$ & 0.20 & $\begin{array}{l}\text { TIR-NBS-LRR class } \\
\text { gene }\end{array}$ \\
\hline \multirow{7}{*}{$2 \mathrm{~B}$} & \multirow{7}{*}{ A03_68863700 } & A03 & BnaA03g43880D & $20.5-23.5$ & 1.30 & $\begin{array}{l}\text { ENHANCED } \\
\text { Disease } \\
\text { RESISTANCE 2-like } \\
\text { isoform X1 }\end{array}$ \\
\hline & & A03 & BnaA03g44070D & $20.5-23.5$ & 0.73 & $\begin{array}{l}\text { TIR-NBS-LRR class } \\
\text { gene }\end{array}$ \\
\hline & & A03 & BnaA03g44080D & $20.5-23.5$ & 0.73 & $\begin{array}{l}\text { TIR-NBS-LRR class } \\
\text { gene }\end{array}$ \\
\hline & & A03 & BnaA03g44090D & $20.5-23.5$ & 0.73 & $\begin{array}{l}\text { Disease resistance } \\
\text { RRS1-like isoform } \\
\mathrm{X} 1\end{array}$ \\
\hline & & A03 & BnaA03g45000D & $20.5-23.5$ & 0.13 & $\begin{array}{l}\text { TIR-NBS-LRR class } \\
\text { gene }\end{array}$ \\
\hline & & A03 & BnaA03g45010D & $20.5-23.5$ & 0.12 & $\begin{array}{l}\text { TIR-NBS-LRR class } \\
\text { gene }\end{array}$ \\
\hline & & A03 & BnaA03g45020D & $20.5-23.5$ & 0.12 & $\begin{array}{l}\text { TIR-NBS-LRR class } \\
\text { gene }\end{array}$ \\
\hline \multirow[t]{3}{*}{ 3D } & \multirow[t]{3}{*}{ A03_71057307 } & A03 & BnaA03g45040D & $20.5-23.5$ & 0.11 & $\begin{array}{l}\text { TIR-NBS-LRR class } \\
\text { gene }\end{array}$ \\
\hline & & A03 & BnaA03g45050D & $20.5-23.5$ & 0.11 & $\begin{array}{l}\text { TIR-NBS-LRR class } \\
\text { gene }\end{array}$ \\
\hline & & A03 & BnaA03g45970D & $20.5-23.5$ & 0.46 & $\begin{array}{l}\text { TIR-NBS-LRR class } \\
\text { gene }\end{array}$ \\
\hline \multirow{5}{*}{$2 B$} & \multirow{5}{*}{ A04_83864566 } & A04 & BnaA04g06520D & $5.5-6.5$ & 0.92 & $\begin{array}{l}\text { Putative Disease } \\
\text { resistance protein }\end{array}$ \\
\hline & & A04 & BnaA04g06530D & $5.5-6.5$ & 0.92 & $\begin{array}{l}\text { Putative Disease } \\
\text { resistance protein }\end{array}$ \\
\hline & & A04 & BnaA04g06550D & $5.5-6.5$ & 0.91 & $\begin{array}{l}\text { Putative Disease } \\
\text { resistance protein }\end{array}$ \\
\hline & & A04 & BnaA04g06580D & $5.5-6.5$ & 0.82 & $\begin{array}{l}\text { Putative Disease } \\
\text { resistance protein }\end{array}$ \\
\hline & & A04 & BnaA04g06780D & $5.5-6.5$ & 0.63 & $\begin{array}{l}\text { Disease resistance- } \\
\text { responsive } \\
\text { (dirigent-like } \\
\text { protein) }\end{array}$ \\
\hline $2 \mathrm{~B}$ & A05_115772286 & A05 & BnaA05g24990D & $18-19$ & 0.22 & $\begin{array}{l}\text { CC-NBS-LRR class } \\
\text { gene }\end{array}$ \\
\hline
\end{tabular}




\begin{tabular}{|c|c|c|c|c|c|c|}
\hline Pathotype & SNP & Chromosome & Gene ID & $\begin{array}{l}\text { Candidate } \\
\text { gene } \\
\text { region } \\
(\mathrm{Mb})\end{array}$ & $\begin{array}{l}\text { Distance } \\
\text { to SNP } \\
(\mathrm{Mb})\end{array}$ & Description \\
\hline & & A05 & BnaA05g25000D & $18-19$ & 0.21 & $\begin{array}{l}\text { CC-NBS-LRR class } \\
\text { gene }\end{array}$ \\
\hline $5 X$ & A08_171171159 & A08 & BnaA08g02210D & $1-3$ & 0.94 & $\begin{array}{l}\text { CC-NBS-LRR class } \\
\text { gene }\end{array}$ \\
\hline \multirow{6}{*}{$2 \mathrm{~B}$} & \multirow{5}{*}{ A08_186203638 } & A08 & BnaA08g24820D & $17-18$ & 0.52 & $\begin{array}{l}\text { CC-NBS-LRR class } \\
\text { gene }\end{array}$ \\
\hline & & A08 & BnaA08g24860D & $17-18$ & 0.49 & $\begin{array}{l}\text { CC-NBS-LRR class } \\
\text { gene }\end{array}$ \\
\hline & & A08 & BnaA08g26370D & $17-18$ & 0.12 & $\begin{array}{l}\text { CC-NBS-LRR class } \\
\text { gene }\end{array}$ \\
\hline & & A08 & BnaA08g26380D & $17-18$ & 0.13 & $\begin{array}{l}\text { CC-NBS-LRR class } \\
\text { gene }\end{array}$ \\
\hline & & A08 & BnaA08g26400D & $17-18$ & 0.14 & $\begin{array}{l}\text { CC-NBS-LRR class } \\
\text { gene }\end{array}$ \\
\hline & A08_186203638 & A08 & BnaA08g26410D & $17-18$ & 0.14 & $\begin{array}{l}\text { CC-NBS-LRR class } \\
\text { gene }\end{array}$ \\
\hline \multirow{10}{*}{$5 X$} & \multirow{10}{*}{ A09_195497763 } & A09 & BnaA09g13280D & $7-9$ & 0.76 & $\begin{array}{l}\text { TIR-NBS-LRR class } \\
\text { gene }\end{array}$ \\
\hline & & A09 & BnaA09g13850D & $7-9$ & 0.20 & $\begin{array}{l}\text { TIR-NBS-LRR class } \\
\text { gene }\end{array}$ \\
\hline & & A09 & BnaA09g13890D & $7-9$ & 0.16 & $\begin{array}{l}\text { TIR-NBS-LRR class } \\
\text { gene }\end{array}$ \\
\hline & & A09 & BnaA09g13900D & $7-9$ & 0.15 & $\begin{array}{l}\text { TIR-NBS-LRR class } \\
\text { gene }\end{array}$ \\
\hline & & A09 & BnaA09g14320D & $7-9$ & 0.11 & $\begin{array}{l}\text { TIR-NBS-LRR class } \\
\text { gene }\end{array}$ \\
\hline & & A09 & BnaA09g14420D & $7-9$ & 8.26 & $\begin{array}{l}\text { CC-NBS-LRR class } \\
\text { gene }\end{array}$ \\
\hline & & A09 & BnaA09g14550D & $7-9$ & 0.30 & $\begin{array}{l}\text { CC-NBS-LRR class } \\
\text { gene }\end{array}$ \\
\hline & & A09 & BnaA09g14560D & $7-9$ & 0.30 & $\begin{array}{l}\text { CC-NBS-LRR class } \\
\text { gene }\end{array}$ \\
\hline & & A09 & BnaA09g14570D & $7-9$ & 0.31 & $\begin{array}{l}\text { CC-NBS-LRR class } \\
\text { gene }\end{array}$ \\
\hline & & A09 & BnaA09g14680D & $7-9$ & 0.37 & $\begin{array}{l}\text { CC-NBS-LRR class } \\
\text { gene }\end{array}$ \\
\hline & & A09 & BnaA09g42680D & $27.1-30.1$ & 1.28 & $\begin{array}{l}\text { CC-NBS-LRR class } \\
\text { gene }\end{array}$ \\
\hline & & A09 & BnaA09g43420D & $27.1-30.1$ & 1.66 & $\begin{array}{l}\text { Disease resistance- } \\
\text { responsive } \\
\text { (dirigent-like } \\
\text { protein) family }\end{array}$ \\
\hline $2 B$ & A09_215839211 & A09 & BnaA09g43430D & 27.1-30.1 & 1.67 & $\begin{array}{l}\text { Disease resistance- } \\
\text { responsive } \\
\text { (dirigent-like } \\
\text { protein) family }\end{array}$ \\
\hline
\end{tabular}




\begin{tabular}{|c|c|c|c|c|c|c|}
\hline Pathotype & SNP & Chromosome & Gene ID & $\begin{array}{l}\text { Candidate } \\
\text { gene } \\
\text { region } \\
(\mathrm{Mb})\end{array}$ & $\begin{array}{l}\text { Distance } \\
\text { to SNP } \\
(\mathrm{Mb})\end{array}$ & Description \\
\hline & & A09 & BnaA09g39390D & $27.1-30.1$ & 0.51 & $\begin{array}{l}\text { ENHANCED } \\
\text { Disease }\end{array}$ \\
\hline & & A09 & BnaA09g39400D & $27.1-30.1$ & 0.51 & $\begin{array}{l}\text { RESISTANCE 4-like } \\
\text { ENHANCED } \\
\text { Disease } \\
\text { RESISTANCE 4-like }\end{array}$ \\
\hline & & A10 & BnaA10g04000D & $1-3$ & 0.98 & $\begin{array}{l}\text { ENHANCED } \\
\text { DISEASE } \\
\text { RESISTANCE-like } \\
\text { protein (DUF1336) }\end{array}$ \\
\hline $3 \mathrm{~A}$ & A10_222415846 & A10 & BnaA10g05000D & $1-3$ & 1.60 & $\begin{array}{l}\text { CC-NBS-LRR class } \\
\text { gene }\end{array}$ \\
\hline \multirow{6}{*}{$3 \mathrm{~A}$} & \multirow{6}{*}{ C01_276968702 } & $\mathrm{C} 01$ & BnaC01g39050D & $37-39$ & 0.46 & $\begin{array}{l}\text { Disease resistance } \\
\text { protein RPS6 } \\
\text { isoform X1 }\end{array}$ \\
\hline & & $\mathrm{C} 01$ & BnaC01g40270D & $37-39$ & 0.36 & $\begin{array}{l}\text { TIR-NBS-LRR class } \\
\text { gene }\end{array}$ \\
\hline & & $\mathrm{C} 01$ & BnaC01g40280D & 37-39 & 0.36 & $\begin{array}{l}\text { TIR-NBS-LRR class } \\
\text { gene }\end{array}$ \\
\hline & & C01 & BnaC01g40300D & $37-39$ & 0.37 & $\begin{array}{l}\text { TIR-NBS-LRR class } \\
\text { gene }\end{array}$ \\
\hline & & $\mathrm{C} 01$ & BnaC01g40310D & $37-39$ & 0.38 & $\begin{array}{l}\text { TIR-NBS-LRR class } \\
\text { gene }\end{array}$ \\
\hline & & $\mathrm{C} 01$ & BnaC01g40460D & $37-39$ & 0.44 & $\begin{array}{l}\text { Putative Disease } \\
\text { resistance protein } \\
\text { At } 4 \mathrm{~g} 11170\end{array}$ \\
\hline 2B & C03_685453245 & $\mathrm{C} 03$ & BnaC03g04690D & $2-3$ & 0.48 & $\begin{array}{l}\text { TIR-NBS-LRR class } \\
\text { gene }\end{array}$ \\
\hline $3 \mathrm{D}$ & C03_688995474 & C03 & BnaC03g05380D & $2-3$ & 0.16 & $\begin{array}{l}\text { TIR-NBS-LRR class } \\
\text { gene }\end{array}$ \\
\hline $3 \mathrm{~A}$ & C04_403341747 & $\mathrm{C} 04$ & BnaC04g18730D & $17-19$ & 0.48 & $\begin{array}{l}\text { CC-NBS-LRR class } \\
\text { gene }\end{array}$ \\
\hline $5 \mathrm{X}$ & C08_579178095 & $\mathrm{C08}$ & BnaC08g17450D & $20.7-21.1$ & 0.31 & $\begin{array}{l}\text { TIR-NBS-LRR class } \\
\text { gene }\end{array}$ \\
\hline \multirow{5}{*}{$3 \mathrm{~A}$} & \multirow{5}{*}{ C09_608174205 } & C09 & BnaC09g14400D & $11-12$ & 0.26 & $\begin{array}{l}\text { TIR-NBS-LRR class } \\
\text { gene }\end{array}$ \\
\hline & & C09 & BnaC09g14870D & $11-12$ & 0.12 & $\begin{array}{l}\text { TIR-NBS-LRR class } \\
\text { gene }\end{array}$ \\
\hline & & C09 & BnaC09g15010D & $11-12$ & 0.24 & $\begin{array}{l}\text { CC-NBS-LRR class } \\
\text { gene }\end{array}$ \\
\hline & & C09 & BnaC09g15020D & $11-12$ & 0.24 & $\begin{array}{l}\text { CC-NBS-LRR class } \\
\text { gene }\end{array}$ \\
\hline & & C09 & BnaC09g15110D & $11-12$ & 0.39 & $\begin{array}{l}\text { CC-NBS-LRR class } \\
\text { gene }\end{array}$ \\
\hline $3 \mathrm{D}$ & A09_638459286 & C09 & BnaC09g38250D & $41-42$ & 0.38 & Disease resistance \\
\hline
\end{tabular}


Figures
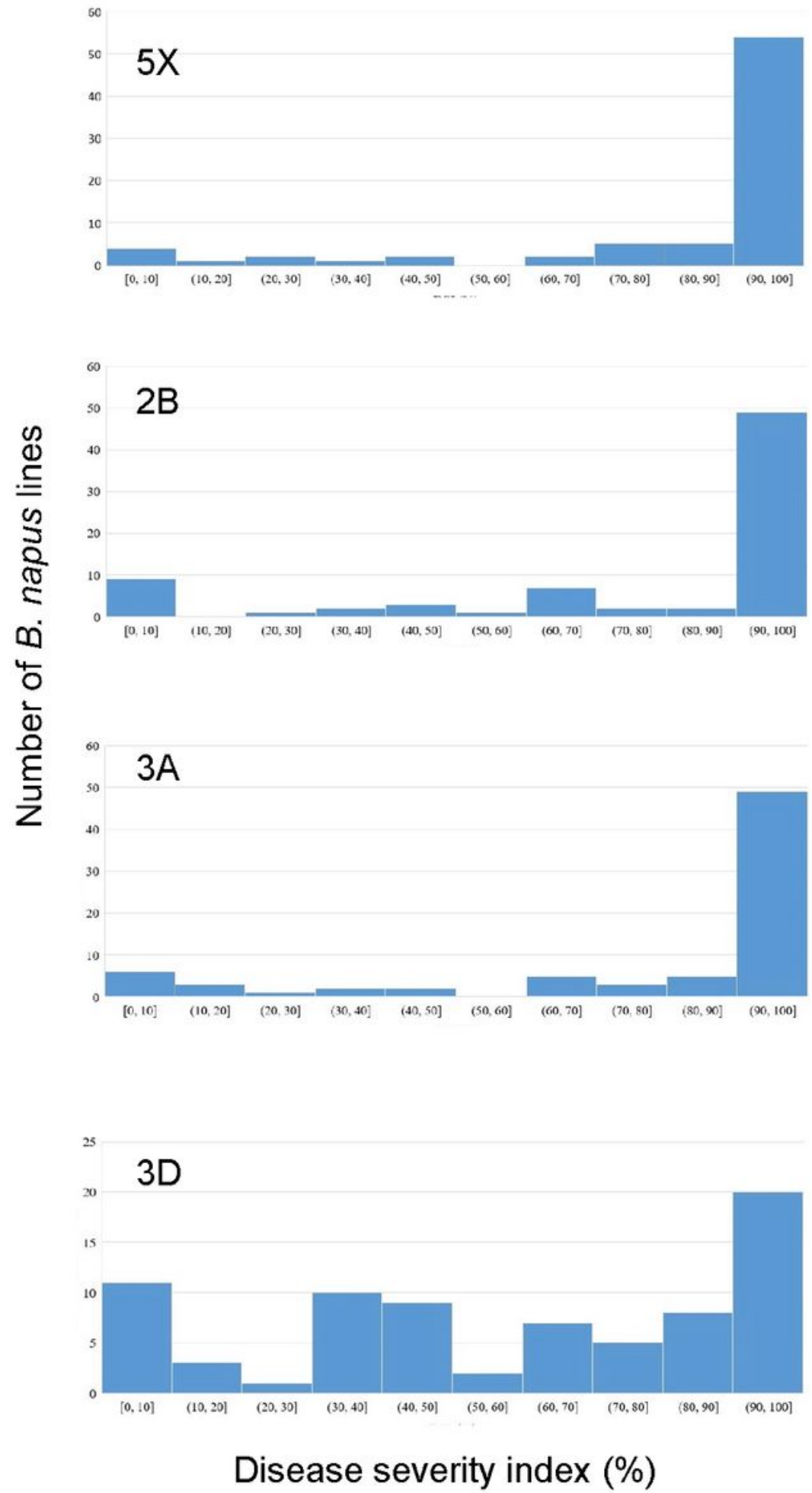

\section{Figure 1}

Frequency distribution of accessions plotted against clubroot severity (disease severity index, DSI) for four pathotypes $5 \mathrm{X}, 2 \mathrm{~B}, 3 \mathrm{~A}$, and $3 \mathrm{D}$ indicated in the figure. 

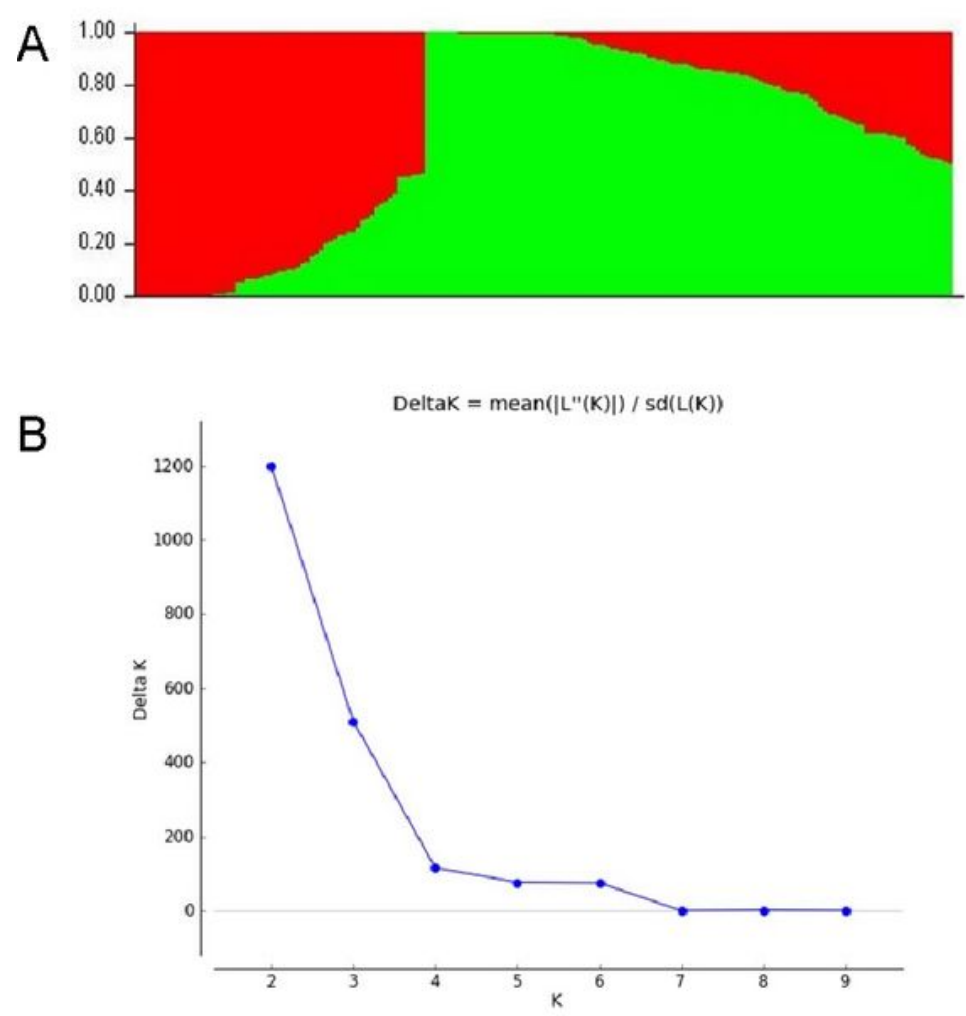

\section{Figure 2}

Population structure analysis of the 177 accessions based on A. model-based Bayesian clustering using STRUCTURE for K = 2 groups, and B. estimation of the number of sub-populations for $\mathrm{K}$ values of 1 to 10 . 
A
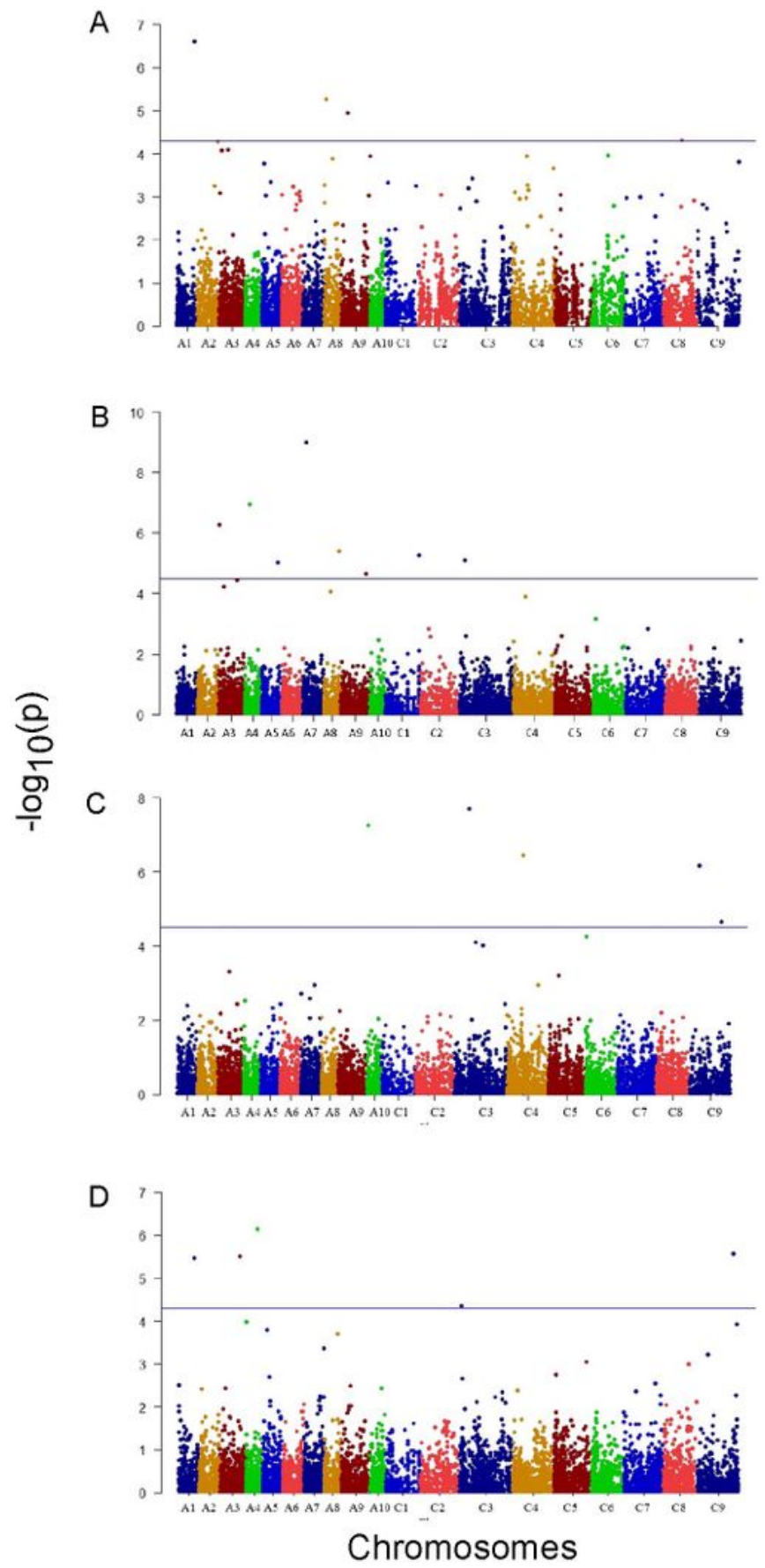

\section{Figure 3}

Manhattan plots of association analysis using the multilocus mixed linear model (MMLM) model $\mathrm{P}+\mathrm{K}$ for pathotypes A. 5X, B. 2B, C. 3A and D. 3D. The horizontal line represents the threshold of significance ($\log 100.5 / 10094=4.30)$.

\section{Supplementary Files}


This is a list of supplementary files associated with this preprint. Click to download.

- eq1.jpg

- Additionalfilesfinalversion.docx 